\title{
A High Order Theory for Linear Thermoelastic Shells: Comparison with Classical Theories
}

\author{
V. V. Zozulya \\ Centro de Investigacion Cientifica de Yucatan, A.C., Calle 43 No. 130, Colonia Chuburná de Hidalgo, \\ 97200 Mérida, YUC, Mexico \\ Correspondence should be addressed to V. V. Zozulya; zozulya@cicy.mx
}

Received 13 August 2012; Accepted 22 January 2013

Academic Editor: Radhey S. Jangid

Copyright (c) 2013 V. V. Zozulya. This is an open access article distributed under the Creative Commons Attribution License, which permits unrestricted use, distribution, and reproduction in any medium, provided the original work is properly cited.

\begin{abstract}
A high order theory for linear thermoelasticity and heat conductivity of shells has been developed. The proposed theory is based on expansion of the 3D equations of theory of thermoelasticity and heat conductivity into Fourier series in terms of Legendre polynomials. The first physical quantities that describe thermodynamic state have been expanded into Fourier series in terms of Legendre polynomials with respect to a thickness coordinate. Thereby all equations of elasticity and heat conductivity including generalized Hooke's and Fourier's laws have been transformed to the corresponding equations for coefficients of the polynomial expansion. Then in the same way as in the 3-D theories system of differential equations in terms of displacements and boundary conditions for Fourier coefficients has been obtained. First approximation theory is considered in more detail. The obtained equations for the first approximation theory are compared with the corresponding equations for Timoshenko's and KirchhoffLove's theories. Special case of plates and cylindrical shell is also considered, and corresponding equations in displacements are presented.
\end{abstract}

\section{Introduction}

The development of microelectromechanical and nanoelectromechanical technologies extends the field of application of the classical or nonclassical theories of plates and shells towards the new thin-walled structures. The classical elasticity can be extended to the micro- and nanoscale by implementation of the theory of elasticity taking into account the physical phenomenon that can occur in such structures and devices $[1,2]$.

Classical theories of beams, rods, plates, and shells are usually related to names of Bernoulli, Euler, Kirchhoff, and Love. These theories are based on well-known physical hypothesis; they are very popular among an engineering community because of their relative simplicity and physical clarity. Numerous books and monographs have been written in the subject among others one can refer to [3-6]. But unfortunately classical theories have some shortcomings and logical contradictions such as their proximity and inaccuracy and as result in some cases not good agreement with results obtained with 3D approach and experiments. Therefore there is demand in developing new more accurate theories.

We can mention at least two approaches to development of the theories of thin-walled structures. One consists in improvement of the classical physical hypothesis and development of more accurate theories. The theory of beamis wellknown model that takes into account transversal deformations developed by Timoshenko and extended to the plate theory by Mindlin [7]. This theory was extended and applied to shells of arbitrary geometry in numerous publications and is referred to as Timoshenko's theory that takes into account in-plane shear deformations and rotation of the elements perpendicular to the middle surface of the shell [8-10].

The second approach consists in expansion of the stressstrain field components into polynomials series in terms of thickness. It was proposed by Cauchy and Poisson and at that time was not popular. Significant extension and development of that method were done by Kil'chevskii [11]. Vekua has used Legendre's polynomials for the expansion of the equations of elasticity and reduction of the 3-D problem to 2-D one 
[12]. Such an approach has significant advantages because Legendre's polynomials are orthogonal, and as a result obtained equations are simpler. This approach was extended and applied to dynamical problems [13], thermoelasticity [14], and composite and laminate shells [15].

The approach developed in [12-15] has been applied to the plates and shells thermoelastic contact problems when mechanical and thermal conditions are changed during deformation in our previous publications [16-29]. The mathematical formulation, differential equations, and contact conditions for the cases of plates and cylindrical shells for the first time have been reported in [16-18]. In more general form with extension to nonstationary processes and calculation of all coefficients of the equations and contact and boundary conditions it was presented in $[19,20]$. Then the approach was further developed to contact of plates and shells with rigid bodies through heat conducting layer [21-25], thermoelasticity of the laminated composite materials with possibility of delamination and mechanical and thermal contact in temperature field in $[21,22,26]$, the pencil-thin nuclear fuel rods modeling in [22], and functionally graded shells in $[28,29]$.

Bibliography related to different aspects of the theory and applications of the thin-walled structures contains of several thousands publications for references one can see review papers [30, 31]. For trends and recent development in the shells theory and its applications one can refer to books [10, 32-34].

In this paper, an approach based on expansion of the equations of thermoelasticity and heat conductivity into Fourier series in terms of Legendre polynomials has been developed and applied to high order theory of arbitrary geometry shells. For that purpose we expand functions that describe thermodynamic state of elastic body into Fourier series in terms of Legendre polynomials with respect to thickness and find corresponding relations of thermoelasticity and heat conductivity for Fourier coefficients of those. Then using techniques developed in our previous publications we find system of differential equations and boundary conditions for Fourier coefficients. Case of first approximation (Vekua's) theory is considered in more detail, and all relations and equations are explicitly presented. Obtained equations for Vekua's shell theory have been analyzed and compared with classical Timoshenko's and Kirchhoff-Love's shell theories.

\section{3-D Formulation}

Let an elastic body occupy an open 3-D Euclidian space in simply connected bounded domain $V \in \mathbf{R}^{3}$ with a smooth boundary $\partial V$. We assume that body is homogeneous isotropic shell of arbitrary geometry with $2 h$ thickness, material of which follows linear physical laws of thermoelasticity and heat conductivity. The shell occupies the domain $V=\Omega \times$ $[-h, h]$ in Euclidean space. Boundary of the shell can be presented in the form $\partial V=S \cup \Omega^{+} \cup \Omega^{-}$. Here $\Omega$ is the middle surface of the shell, $\partial \Omega$ is its boundary, $\Omega^{+}$and $\Omega^{-}$are the outer sides, and $S=\partial \Omega \times[-h, h]$ is a sheer side.
Thermodynamic state of the body is defined by stress $\sigma_{i j}(\mathbf{x}, t)$ and strain $\varepsilon_{i j}(\mathbf{x}, t)$ tensors and displacements $u_{i}(\mathbf{x}, t)$, traction $p_{i}(\mathbf{x}, t)$, and body forces $b_{i}(\mathbf{x}, t)$ vectors, temperature $\theta(\mathbf{x}, t)$, and vector of thermal flow $q_{i}(\mathbf{x}, t)$ and specific strength of the internal heat sources $\chi(\mathbf{x}, t)$, respectively. These quantities are not independent they are related by equations of linear thermoelasticity and heat conductivity.

We introduce orthogonal system of coordinates $x_{1}, x_{2}$, and $x_{3}$, such that position vector of arbitrary point is equal to $\mathbf{R}\left(x_{1}, x_{2}, x_{3}\right)=\mathbf{e}_{i} x_{i}$. Unit orthogonal basic vectors and their derivatives with respect to space coordinates are equal to

$$
\mathbf{e}_{i}=\frac{1}{H_{i}} \frac{\partial \mathbf{R}}{\partial x_{i}}, \quad \frac{\partial \mathbf{e}_{i}}{\partial x_{j}}=\Gamma_{i j}^{k} \mathbf{e}_{k},
$$

where $H_{i}$ are Lame coefficients and $\Gamma_{i j}^{k}$ are Christoffel symbols. They are calculated by the equations

$$
\begin{gathered}
H_{i}=\left|\frac{\partial \mathbf{R}}{\partial x_{i}}\right|=\sqrt{\frac{\partial \mathbf{R}}{\partial x_{i}} \cdot \frac{\partial \mathbf{R}}{\partial x_{i}}}, \\
\Gamma_{i j}^{k}=-\frac{1}{H_{i}} \frac{\partial H_{i}}{\partial x_{j}} \delta_{i k}+\frac{1}{2 H_{i} H_{k}} \\
\times\left(\delta_{j k} \frac{\partial H_{j} H_{k}}{\partial x_{i}}+\delta_{i k} \frac{\partial H_{i} H_{k}}{\partial x_{j}}-\delta_{i j} \frac{\partial H_{i} H_{j}}{\partial x_{k}}\right) .
\end{gathered}
$$

From the last equation it follows that $\Gamma_{i j}^{k}=0$ for $i \neq j \neq k$ and

$$
\Gamma_{i i}^{k}=-\frac{1}{H_{k}} \frac{\partial H_{i}}{\partial x_{k}}, \quad \Gamma_{i k}^{k}=-\frac{1}{H_{i}} \frac{\partial H_{k}}{\partial x_{i}} \quad \text { for } i \neq k .
$$

In the case if displacements and their gradients are small, the following kinematic Cauchy relations take place:

$$
\varepsilon_{i j}=\frac{1}{2}\left(\frac{1}{H_{j}} \frac{\partial u_{i}}{\partial x_{j}}+\frac{1}{H_{i}} \frac{\partial u_{j}}{\partial x_{i}}\right)+\Gamma_{i j}^{k} u_{k} .
$$

Equations of motion have the form

$$
\frac{1}{H_{j}} \frac{\partial \sigma_{i j}}{\partial x_{j}}+\frac{\sigma_{i j}}{H_{k}} \Gamma_{i k}^{k}+\frac{\sigma_{i k}}{H_{i}} \Gamma_{k i}^{j}+b_{i}=\rho \ddot{u}_{i},
$$

where upper points are partial derivatives with respect to time $t$.

We assume that the stress $\sigma_{i j}(\mathbf{x})$ and deformation $\varepsilon_{i j}(\mathbf{x})$ tensors and temperature $\theta(\mathbf{x}, t)$ are related by equations

$$
\sigma_{i j}=c_{i j k l} \sigma_{k l}+\beta_{i j} \theta, \quad c_{i j k l}=c_{j i k l}=c_{k l i j}, \quad \beta_{i j}=\beta_{j i},
$$

where $c_{i j k l}$ and $\beta_{i j}$ are elastic modulus and the coefficients of linear thermal expansion. In the isotropic case

$$
c_{i j k l}=\lambda \delta_{i j} \delta_{k l}+\mu\left(\delta_{i k} \delta_{j l}+\delta_{i l} \delta_{k j}\right), \quad \beta_{i j}=\beta_{T} \delta_{i j},
$$

where $\lambda$ and $\mu$ are the Lame constants, $\beta_{T}=(\mu+3 \lambda) \alpha_{T}$, and $\alpha_{T}$ is a coefficient of linear thermal expansion. 
Equations (6) and (7) are referred to as generalized Hooke's law. They were introduced by Duhamel and substantiated using thermodynamic methods by Bio $[8,11]$.

Heat is distributed in the body according to Fourie's law:

$$
q_{i}=\lambda_{i j} \frac{1}{H_{j}} \frac{\partial \theta}{\partial x_{j}},
$$

where $\lambda_{i j}$ is the tensor of coefficients of thermal conductivity of the body, in the isotropic case $\lambda_{i j}=\delta_{i j} \lambda_{T}$, and $\lambda_{T}$ is the coefficient of thermal conductivity of the body.

For convenience we transform the previous equations taking into account that the position vector $\mathbf{R}(\mathbf{x})$ of any point in domain $V$, occupied by material points of shell, may be presented as

$$
\mathbf{R}(\mathbf{x})=\mathbf{r}\left(\mathbf{x}_{\alpha}\right)+x_{3} \mathbf{n}\left(\mathbf{x}_{\alpha}\right),
$$

where $\mathbf{r}\left(\mathbf{x}_{\alpha}\right)$ is the position vector of the points located on the middle surface of shell and $\mathbf{n}\left(\mathbf{x}_{\alpha}\right)$ is a unit vector normal to the middle surface.

We consider that $\mathbf{x}_{\alpha}=\left(x^{1}, x^{2}\right)$ are curvilinear coordinates associated with main curvatures of the middle surface of the shell. In this case 3-D equations (4)-(8) can be simplified taking into account that Lame coefficients and their derivatives have the form

$$
\begin{aligned}
H_{\alpha} & =A_{\alpha}\left(1+k_{\alpha} x_{3}\right) \quad \text { for } \alpha=1,2, H_{3}=1 \\
\frac{\partial H_{\beta}}{\partial x_{\alpha}} & =\frac{\partial A_{\beta}}{\partial x_{\alpha}}\left(1+k_{\alpha} x_{3}\right), \quad \frac{\partial H_{\beta}}{\partial x_{3}}=k_{\beta} A_{\beta}, \quad \frac{\partial H_{3}}{\partial x_{i}}=0 .
\end{aligned}
$$
form

The equations of motion (5) after simplification have the

$$
\begin{aligned}
& \frac{\partial\left(A_{2} \sigma_{11}\right)}{\partial x_{1}}+\frac{\partial\left(A_{1} \sigma_{12}\right)}{\partial x_{2}}+A_{1} A_{2} \frac{\partial \sigma_{13}}{\partial x_{3}}+\sigma_{12} \frac{\partial A_{1}}{\partial x_{2}} \\
& +\sigma_{13} A_{1} A_{2} k_{1}-\sigma_{22} \frac{\partial A_{2}}{\partial x_{1}}+A_{1} A_{2} b_{1}=\rho A_{1} A_{2} \ddot{u}_{1}, \\
& \frac{\partial\left(A_{2} \sigma_{21}\right)}{\partial x_{1}}+\frac{\partial\left(A_{1} \sigma_{22}\right)}{\partial x_{2}}+A_{1} A_{2} \frac{\partial \sigma_{23}}{\partial x_{3}}+\sigma_{21} \frac{\partial A_{2}}{\partial x_{1}} \\
& \quad+\sigma_{23} A_{1} A_{2} k_{2}-\sigma_{11} \frac{\partial A_{1}}{\partial x_{2}}+A_{1} A_{2} b_{2}=\rho A_{1} A_{2} \ddot{u}_{2}, \\
& \frac{\partial\left(A_{2} \sigma_{31}\right)}{\partial x_{1}}+\frac{\partial\left(A_{1} \sigma_{32}\right)}{\partial x_{2}}+\frac{\partial\left(A_{1} A_{2} \sigma_{33}\right)}{\partial x_{3}}-\sigma_{11} A_{1} A_{2} k_{1} \\
& \quad-\sigma_{22} A_{1} A_{2} k_{2}+A_{1} A_{2} b_{3}=\rho A_{1} A_{2} \ddot{u}_{3} .
\end{aligned}
$$

The kinematic Cauchy relations (4) have the form

$$
\begin{aligned}
& \varepsilon_{11}=\frac{1}{A_{1}} \frac{\partial u_{1}}{\partial x_{1}}+\frac{1}{A_{1} A_{2}} \frac{\partial A_{1}}{\partial x_{2}} u_{2}+k_{1} u_{3}, \\
& \varepsilon_{22}=\frac{1}{A_{2}} \frac{\partial u_{2}}{\partial x_{2}}+\frac{1}{A_{2} A_{1}} \frac{\partial A_{2}}{\partial x_{1}} u_{1}+k_{2} u_{3},
\end{aligned}
$$

$$
\begin{gathered}
\varepsilon_{33}=\frac{\partial u_{3}}{\partial x_{3}}, \\
\varepsilon_{12}=\frac{1}{A_{2}}\left(\frac{\partial u_{1}}{\partial x_{2}}-\frac{1}{A_{1}} \frac{\partial A_{2}}{\partial x_{1}} u_{2}\right)+\frac{1}{A_{1}}\left(\frac{\partial u_{2}}{\partial x_{1}}-\frac{1}{A_{2}} \frac{\partial A_{1}}{\partial x_{2}} u_{1}\right), \\
\varepsilon_{13}=\frac{\partial u_{1}}{\partial x_{3}}-k_{1} u_{1}+\frac{1}{A_{1}} \frac{\partial u_{3}}{\partial x_{1}} \\
\varepsilon_{23}=\frac{\partial u_{2}}{\partial x_{3}}-k_{2} u_{2}+\frac{1}{A_{2}} \frac{\partial u_{3}}{\partial x_{2}} .
\end{gathered}
$$

In (10)-(12), $A_{\alpha}\left(x_{1}, x_{2}\right)=\left(\partial \mathbf{r}\left(x_{1}, x_{2}\right)\right) / \partial x_{\alpha}$ are coefficients of the first quadratic form of a surface, and $k_{\alpha}\left(x_{1}, x_{2}\right)$ are its main curvatures. We also take into account that shell is relatively thin, and therefore

$$
\frac{1}{H_{\alpha}} \frac{\partial H_{\beta}}{\partial x_{\alpha}}=\frac{1}{A_{\alpha}} \frac{\partial A_{\beta}}{\partial x_{\alpha}}, \quad 1+k_{\alpha} x_{3} \approx 1 \longrightarrow H_{\alpha} \approx A_{\alpha} .
$$

Following $[8,11]$ we can present coupled system of the equations of linear thermoelasticity and heat conductivity in the form

$\mathbf{L} \cdot \mathbf{u}+\beta_{T} \nabla \theta+\mathbf{b}=\rho \ddot{\mathbf{u}}, \quad \forall \mathbf{x} \in V, \quad \forall t \in \mathfrak{I}=[0, T]$,

$$
\lambda_{T} \Delta \theta-\chi=c_{\varepsilon} \dot{\theta}+\beta_{T} \theta_{0}(\nabla \cdot \dot{\mathbf{u}}) .
$$

The differential operator $\mathbf{L}=L_{i j} \mathbf{e}_{i} \otimes \mathbf{e}_{j}$ for homogeneous isotropic medium has the form

$$
\mathbf{L} \cdot \mathbf{u}=\mu \Delta \mathbf{u}+(\lambda+\mu) \nabla(\nabla \cdot \mathbf{u}) .
$$

Other differential operators presented in (14) and (15), respectively, in the system of coordinates introduced here related to middle surface of the shell have the form

$$
\begin{gathered}
\nabla(\mathbf{u})_{1}=\frac{1}{A_{1}} \frac{\partial u_{1}}{\partial x_{1}}+\frac{1}{A_{2}} \frac{\partial u_{1}}{\partial x_{2}}+\frac{\partial u_{1}}{\partial x_{3}}, \\
\nabla(\nabla \cdot \mathbf{u})_{\alpha}=\frac{1}{A_{\alpha}} \frac{\partial}{\partial x_{\alpha}}\left(\frac{1}{A_{1} A_{2}}\left[\frac{\partial\left(A_{2} u_{1}\right)}{\partial x_{1}}+\frac{\partial\left(A_{1} u_{2}\right)}{\partial x_{2}}\right]\right. \\
\left.+\frac{\partial u_{3}}{\partial x_{3}}+\left(k_{1}+k_{2}\right) u_{3}\right), \\
\nabla(\nabla \cdot \mathbf{u})_{3}=\frac{\partial}{\partial x_{3}}\left(\frac{1}{A_{1} A_{2}}\left[\frac{\partial\left(A_{2} u_{1}\right)}{\partial x_{1}}+\frac{\partial\left(A_{1} u_{2}\right)}{\partial x_{2}}\right]\right. \\
\left.+\frac{\partial u_{3}}{\partial x_{3}}+\left(k_{1}+k_{2}\right) u_{3}\right), \\
\nabla \theta=\frac{1}{A_{1}} \frac{\partial \theta}{\partial x_{1}}+\frac{1}{A_{2}} \frac{\partial \theta}{\partial x_{2}}+\frac{\partial \theta}{\partial x_{3}} .
\end{gathered}
$$

For mathematically correct formulation of the coupled problem of thermoelasticity and heat conductivity we have to formulate initial and boundary conditions. Initial conditions 
consist of assignment of the displacements, velocity, and temperature distribution in the initial moment of time. They can be written in the form

$$
\begin{gathered}
u_{i}(\mathbf{x}, 0)=u_{i}^{0}(\mathbf{x}), \quad \partial_{t} u_{i}(\mathbf{x}, 0)=v_{i}^{0}(\mathbf{x}), \\
\theta(\mathbf{x}, 0)=\theta^{0}(\mathbf{x})
\end{gathered}
$$

If the body occupied a finite region $V$ with the boundary $\partial V$, it is necessary to assign boundary conditions. We consider the mixed boundary conditions in the form

$$
\begin{gathered}
u_{i}(\mathbf{x}, t)=u_{i}^{b}(\mathbf{x}, t), \quad \forall \mathbf{x} \in \partial V_{u}, \quad \forall t \in \mathfrak{J}, \\
p_{i}(\mathbf{x}, t)=\sigma_{i j}(\mathbf{x}, t) n_{j}(\mathbf{x})=p_{i}^{b}(\mathbf{x}, t), \quad \forall \mathbf{x} \in \partial V_{p}, \quad \forall t \in \mathfrak{J}, \\
\theta(\mathbf{x}, t)=\theta^{b}(\mathbf{x}, t), \quad \forall \mathbf{x} \in \partial V_{\theta}, \quad \forall t \in \mathfrak{I}, \\
q_{i}(\mathbf{x}, t)=q_{i}^{b}(\mathbf{x}, t), \quad \forall \mathbf{x} \in \partial V_{q}, \quad \forall t \in \mathfrak{I}
\end{gathered}
$$

Now we can transform 3-D equations of thermoelasticity and heat conductivity in 2-D equations using Legendre polynomials series expansion.

\section{2-D Formulation}

We expand the physical parameters, that describe the thermodynamic state of the body, into the Legendre polynomials series along the coordinate $x_{3}$. Such expansion can be done because any function $f(p)$, which is defined in domain $-1 \leq p \leq 1$ and satisfies Dirichlet's conditions (continuous, monotonous, and having finite set of discontinuity points), can be expanded into Legendre's series according to formulas

$$
f(p)=\sum_{k=0}^{\infty} a_{k} P_{k}(p) \quad \text { where } a_{n}=\frac{2 k+1}{2} \int_{-1}^{1} f(p) P_{k}(p) d p
$$

Any function of more than one independent variable can also be expanded into Legendre's series with respect to for example, variable $x_{3} \in[-1,1]$, but first the new normalized variable $\omega=x_{3} / h \in[-1,1]$ has to be introduced. Taking into account (19) we have

$$
\begin{aligned}
& u_{i}(\mathbf{x}, t)=\sum_{k=0}^{\infty} u_{i}^{k}\left(\mathbf{x}_{\alpha}, t\right) P_{k}(\omega), \\
& \sigma_{i j}(\mathbf{x}, t)=\sum_{k=0}^{\infty} \sigma_{i j}^{k}\left(\mathbf{x}_{\alpha}, t\right) P_{k}(\omega), \\
& \varepsilon_{i j}(\mathbf{x}, t)=\sum_{k=0}^{\infty} \varepsilon_{i j}^{k}\left(\mathbf{x}_{\alpha}, t\right) P_{k}(\omega), \\
& \theta(\mathbf{x}, t)=\sum_{k=0}^{\infty} \theta^{k}\left(\mathbf{x}_{\alpha}, t\right) P_{k}(\omega),
\end{aligned}
$$

$$
\begin{aligned}
& u_{i}^{k}\left(\mathbf{x}_{\alpha}, t\right)=\frac{2 k+1}{2 h} \int_{-h}^{h} u_{i}\left(\mathbf{x}_{\alpha}, x_{3}, t\right) P_{k}(\omega) d x_{3}, \\
& \sigma_{i j}^{k}\left(\mathbf{x}_{\alpha}, t\right)=\frac{2 k+1}{2 h} \int_{-h}^{h} \sigma_{i j}\left(\mathbf{x}_{\alpha}, x_{3}, t\right) P_{k}(\omega) d x_{3}, \\
& \varepsilon_{i j}^{k}\left(\mathbf{x}_{\alpha}, t\right)=\frac{2 k+1}{2 h} \int_{-h}^{h} \varepsilon_{i j}\left(\mathbf{x}_{\alpha}, x_{3}, t\right) P_{k}(\omega) d x_{3}, \\
& \theta^{k}\left(\mathbf{x}_{\alpha}, t\right)=\frac{2 k+1}{2 h} \int_{-h}^{h} \theta\left(\mathbf{x}_{\alpha}, x_{3}, t\right) P_{k}(\omega) d x_{3} .
\end{aligned}
$$

The following relations take place for the derivatives with respect to time:

$$
\begin{aligned}
& \frac{2 k+1}{2 h} \int_{-h}^{h} \partial_{t} u_{i}\left(\mathbf{x}_{\alpha}, x_{3}, t\right) P_{k}(\omega) d x_{3}=\partial_{t} u_{i}^{k}\left(\mathbf{x}_{\alpha}, t\right), \\
& \frac{2 k+1}{2 h} \int_{-h}^{h} \partial_{t}^{2} u_{i}\left(\mathbf{x}_{\alpha}, x_{3}, t\right) P_{k}(\omega) d x_{3}=\partial_{t}^{2} u_{i}^{k}\left(\mathbf{x}_{\alpha}, t\right), \\
& \frac{2 k+1}{2 h} \int_{-h}^{h} \partial_{t} \theta\left(\mathbf{x}_{\alpha}, x_{3}, t\right) P_{k}(\omega) d x_{3}=\partial_{t} \theta^{k}\left(\mathbf{x}_{\alpha}, t\right),
\end{aligned}
$$

and for the derivatives with respect to coordinates $\mathbf{x}_{\alpha}$ :

$$
\begin{array}{r}
\frac{2 k+1}{2 h} \int_{-h}^{h} \frac{\partial u_{i}\left(\mathbf{x}_{\alpha}, x_{3}, t\right)}{\partial x_{\alpha}} P_{k}(\omega) d x_{3}=\frac{\partial u_{i}^{k}\left(\mathbf{x}_{\alpha}, t\right)}{\partial x_{\alpha}}, \\
\frac{2 k+1}{2 h} \int_{-h}^{h} \frac{\partial \sigma_{i j}\left(\mathbf{x}_{\alpha}, x_{3}, t\right)}{\partial x_{\alpha}} P_{k}(\omega) d x_{3}=\frac{\partial \sigma_{i j}^{k}\left(\mathbf{x}_{\alpha}, t\right)}{\partial x_{\alpha}}, \\
\frac{2 k+1}{2 h} \int_{-h}^{h} \frac{\partial \theta\left(\mathbf{x}_{\alpha}, x_{3}, t\right)}{\partial x_{\alpha}} P_{k}(\omega) d x_{3}=\frac{\partial u_{i}^{k}\left(\mathbf{x}_{\alpha}, t\right)}{\partial x_{\alpha}},
\end{array}
$$

respectively.

Integration of the derivatives with respect to coordinates $x_{3}$ gives us

$$
\begin{gathered}
\frac{2 k+1}{2 h} \int_{-h}^{h} \frac{\partial u_{i}(\mathbf{x}, t)}{\partial x_{3}} P_{k}(\omega) d x_{3}=\underline{u_{i}^{k}}\left(\mathbf{x}_{\alpha}, t\right), \\
\frac{2 k+1}{2 h} \int_{-h}^{h} \frac{\partial \sigma_{i 3}(\mathbf{x}, t)}{\partial x_{3}} P_{k}(\omega) d x_{3} \\
=\frac{2 k+1}{h}\left[\sigma_{i 3}^{+}\left(\mathbf{x}_{\alpha}, t\right)-(-1)^{k} \sigma_{i 3}^{-}\left(\mathbf{x}_{\alpha}, t\right)\right]-\underline{\sigma_{i 3}^{k}}\left(\mathbf{x}_{\alpha}, t\right), \\
\frac{2 k+1}{2 h} \int_{-h}^{h} \partial_{3} \theta(\mathbf{x}, t) P_{k}(\omega) d x_{3}=\partial_{3} \theta\left(\mathbf{x}_{\alpha}, t\right)=Q_{3}^{k}\left(\mathbf{x}_{\alpha}, t\right), \\
\frac{2 k+1}{2 h} \int_{-h}^{h} \partial_{3}^{2} \theta(\mathbf{x}, t) P_{k}(\omega) d x_{3} \\
=\frac{2 k+1}{h}\left[Q_{3}^{+}\left(\mathbf{x}_{\alpha}, t\right)-(-1)^{k} Q_{3}^{-}\left(\mathbf{x}_{\alpha}, t\right)\right]+\underline{Q_{3}^{k}}\left(\mathbf{x}_{\alpha}, t\right),
\end{gathered}
$$


where

$$
\begin{gathered}
\underline{u_{i}^{k}}\left(\mathbf{x}_{\alpha}, t\right)=\frac{2 k+1}{h}\left(u_{i}^{k+1}\left(\mathbf{x}_{\alpha}, t\right)+u_{i}^{k+3}\left(\mathbf{x}_{\alpha}, t\right)+\cdots\right), \\
\underline{\sigma_{i 3}^{k}}\left(\mathbf{x}_{\alpha}, t\right)=A_{1} A_{2} \frac{2 k+1}{h}\left(\sigma_{i 3}^{k-1}\left(\mathbf{x}_{\alpha}, t\right)+\sigma_{i 3}^{k-3}\left(\mathbf{x}_{\alpha}, t\right)+\cdots\right), \\
\underline{Q_{3}^{k}}\left(\mathbf{x}_{\alpha}, t\right)=-A_{1} A_{2} \frac{2 k+1}{h}\left(Q_{3}^{k-1}\left(\mathbf{x}_{\alpha}, t\right)+Q_{3}^{k-3}\left(\mathbf{x}_{\alpha}, t\right)+\cdots\right) .
\end{gathered}
$$

Now substituting stress tensor from (20) with considering the equations of motion (11), multiplying obtained relations by $P_{k}(\omega)$, and integrating over interval $[-h, h]$ with respect to $x_{3}$ we obtain 2-D equations of motion in the form

$$
\begin{aligned}
& \frac{\partial\left(A_{2} \sigma_{11}^{k}\right)}{\partial x_{1}}+\frac{\partial\left(A_{1} \sigma_{12}^{k}\right)}{\partial x_{2}}+\sigma_{12}^{k} \frac{\partial A_{1}}{\partial x_{2}}+\sigma_{13}^{k} A_{1} A_{2} k_{1}-\sigma_{22}^{k} \frac{\partial A_{2}}{\partial x_{1}} \\
& \quad-\underline{\sigma_{13}^{k}}+A_{1} A_{2} f_{1}^{k}=\rho A_{1} A_{2} \partial_{t}^{2} u_{1}^{k}, \\
& \frac{\partial\left(A_{2} \sigma_{21}^{k}\right)}{\partial x_{1}}+\frac{\partial\left(A_{1} \sigma_{22}^{k}\right)}{\partial x_{2}}+\sigma_{12}^{k} \frac{\partial A_{2}}{\partial x_{1}}+\sigma_{23}^{k} A_{1} A_{2} k_{2}-\sigma_{11}^{k} \frac{\partial A_{2}}{\partial x_{1}} \\
& -\underline{\sigma_{23}^{k}}+A_{1} A_{2} f_{2}^{k}=\rho A_{1} A_{2} \partial_{t}^{2} u_{2}^{k}, \\
& \frac{\partial\left(A_{2} \sigma_{31}^{k}\right)}{\partial x_{1}}+\frac{\partial\left(A_{1} \sigma_{32}^{k}\right)}{\partial x_{2}}-\sigma_{11}^{k} A_{1} A_{2} k_{1}-\sigma_{22}^{k} A_{1} A_{2} k_{2} \\
& -\underline{\sigma_{33}^{k}}+A_{1} A_{2} f_{3}^{k}=\rho A_{1} A_{2} \partial_{t}^{2} u_{3}^{k},
\end{aligned}
$$

where

$$
f_{i}^{k}\left(\mathbf{x}_{\alpha}\right)=b_{i}^{k}\left(\mathbf{x}_{\alpha}\right)+\frac{2 k+1}{h}\left(\sigma_{i 3}^{+}\left(\mathbf{x}_{\alpha}\right)-(-1)^{k} \sigma_{i 3}^{-}\left(\mathbf{x}_{\alpha}\right)\right) .
$$

In the same way the 2-D kinematic Cauchy relations can be found:

$$
\begin{gathered}
\varepsilon_{11}^{k}=\frac{1}{A_{1}} \frac{\partial u_{1}^{k}}{\partial x_{1}}+\frac{1}{A_{1} A_{2}} \frac{\partial A_{1}}{\partial x_{2}} u_{2}^{k}+k_{1} u_{3}^{k}, \\
\varepsilon_{22}^{k}=\frac{1}{A_{2}} \frac{\partial u_{2}^{k}}{\partial x_{2}}+\frac{1}{A_{1} A_{2}} \frac{\partial A_{2}}{\partial x_{1}} u_{1}^{k}+k_{2} u_{3}^{k}, \\
\varepsilon_{12}^{k}=\frac{1}{A_{2}}\left(\frac{\partial u_{1}^{k}}{\partial x_{2}}-\frac{1}{A_{1}} \frac{\partial A_{2}}{\partial x_{1}} u_{2}^{k}\right)+\frac{1}{A_{1}}\left(\frac{\partial u_{2}^{k}}{\partial x_{1}}-\frac{1}{A_{2}} \frac{\partial A_{1}}{\partial x_{2}} u_{1}^{k}\right), \\
\varepsilon_{13}^{k}=\frac{1}{A_{1}} \frac{\partial u_{3}^{k}}{\partial x_{1}}-k_{1} u_{1}^{k}+\underline{u_{1}^{k}}, \\
\varepsilon_{23}^{k}=\frac{1}{A_{2}} \frac{\partial u_{3}^{k}}{\partial x_{2}}-k_{2} u_{2}^{k}+\underline{u_{2}^{k}}, \quad \varepsilon_{33}=\underline{u_{3}^{k}} .
\end{gathered}
$$

Let us consider generalized Hooke's law for homogeneous anisotropic body

$$
\sigma_{i j}^{k}=c_{i j k l} \varepsilon_{k l}^{k}+\beta_{i j} \theta^{k}
$$

and for isotropic one

$$
\begin{gathered}
\sigma_{11}^{k}=(\lambda+2 \mu) \varepsilon_{11}^{k}+\lambda\left(\varepsilon_{22}^{k}+\varepsilon_{33}^{k}\right)+\beta_{T} \theta^{k}, \\
\sigma_{22}^{k}=(\lambda+2 \mu) \varepsilon_{22}^{k}+\lambda\left(\varepsilon_{11}^{k}+\varepsilon_{33}^{k}\right)+\beta_{T} \theta^{k}, \\
\sigma_{33}^{k}=(\lambda+2 \mu) \varepsilon_{22}^{k}+\lambda\left(\varepsilon_{11}^{k}+\varepsilon_{22}^{k}\right)+\beta_{T} \theta^{k}, \\
\sigma_{12}^{k}=\mu \varepsilon_{12}^{k}, \quad \sigma_{13}^{k}=\mu \varepsilon_{13}^{k}, \\
\sigma_{23}^{k}=\mu \varepsilon_{23}^{k} .
\end{gathered}
$$

In the previously mentioned relations (26)-(30) the following orthogonality property of the Legendre's polynomials has been used:

$$
\int_{-h}^{h} P_{n}(\omega) P_{m}(\omega) d x_{3}= \begin{cases}\frac{2 h}{2 n+1} & \text { for } n=m \\ 0 & \text { for } n \neq m\end{cases}
$$

In order to find 2-D differential equations in the form of displacements we substitute the kinematic Cauchy relations (28) with Hooke's law for homogeneous body (30). As a result we have

$$
\begin{aligned}
& \sigma_{11}^{k}=(\lambda+2 \mu)\left(\frac{1}{A_{1}} \frac{\partial u_{1}^{k}}{\partial x_{1}}+\frac{1}{A_{1} A_{2}} \frac{\partial A_{1}}{\partial x_{2}} u_{2}^{k}+k_{1} u_{3}^{k}\right) \\
& +\lambda\left(\frac{1}{A_{2}} \frac{\partial u_{2}^{k}}{\partial x_{2}}+\frac{1}{A_{1} A_{2}} \frac{\partial A_{2}}{\partial x_{1}} u_{1}^{k}+k_{2} u_{3}^{k}+\underline{u_{3}^{k}}\right)+\beta_{T} \theta^{k} \\
& \sigma_{22}^{k}=(\lambda+2 \mu)\left(\frac{1}{A_{2}} \frac{\partial u_{2}^{k}}{\partial x_{2}}+\frac{1}{A_{1} A_{2}} \frac{\partial A_{2}}{\partial x_{1}} u_{1}^{k}+k_{2} u_{3}^{k}\right) \\
& +\lambda\left(\frac{1}{A_{1}} \frac{\partial u_{1}^{k}}{\partial x_{1}}+\frac{1}{A_{1} A_{2}} \frac{\partial A_{1}}{\partial x_{2}} u_{2}^{k}+k_{1} u_{3}^{k}+\underline{u_{3}^{k}}\right)+\beta_{T} \theta^{k}, \\
& \sigma_{33}^{k}=(\lambda+2 \mu) u_{3}^{k} \\
& +\lambda\left(\left(\frac{1}{A_{1}} \frac{\partial u_{1}^{k}}{\partial x_{1}}+\frac{1}{A_{1} A_{2}} \frac{\partial A_{1}}{\partial x_{2}} u_{2}^{k}+k_{1} u_{3}^{k}\right)\right. \\
& \left.+\left(\frac{1}{A_{2}} \frac{\partial u_{2}^{k}}{\partial x_{2}}+\frac{1}{A_{1} A_{2}} \frac{\partial A_{2}}{\partial x_{1}} u_{1}^{k}\right)\right)+\beta_{T} \theta^{k} \\
& \sigma_{12}^{k}=\mu\left(\frac{1}{A_{2}}\left(\frac{\partial u_{1}^{k}}{\partial x_{2}}-\frac{1}{A_{1}} \frac{\partial A_{2}}{\partial x_{1}} u_{2}^{k}\right)\right. \\
& \left.+\frac{1}{A_{1}}\left(\frac{\partial u_{2}^{k}}{\partial x_{1}}-\frac{1}{A_{2}} \frac{\partial A_{1}}{\partial x_{2}} u_{1}^{k}\right)\right), \\
& \sigma_{13}^{k}=\mu\left(\frac{1}{A_{1}} \frac{\partial u_{3}^{k}}{\partial x_{1}}-k_{1} u_{1}^{k}+\underline{u_{1}^{k}}\right) \text {, } \\
& \sigma_{23}^{k}=\mu\left(\frac{1}{A_{2}} \frac{\partial u_{3}^{k}}{\partial x_{2}}-k_{2} u_{2}^{k}+\underline{u_{2}^{k}}\right) \text {. }
\end{aligned}
$$


Substitution of these equations in the equations of equilibrium (26) gives us the 2-D equations in displacements in the form

$$
\begin{gathered}
\sum_{l=0}^{\infty} L_{i j}^{k l} u_{j}^{l}-\underline{\sigma_{i 3}^{k}}+L_{i}^{k} \theta^{k}+A_{1} A_{2} f_{i}^{k}=\rho A_{1} A_{2} \ddot{u}_{i}^{k}, \\
\lambda_{T} \Delta \theta^{k}+\underline{\underline{Q_{3}^{k}}}+\chi^{k} \\
=c_{\varepsilon} \dot{\theta}^{k}+\beta_{T} \theta_{0}\left[\nabla \cdot \dot{\mathbf{u}}^{k}+\frac{2 k+1}{h}\left(\dot{u}_{3}^{k+1}+\dot{u}_{3}^{k+3} \cdots\right)\right],
\end{gathered}
$$

where

$$
\underline{\underline{Q_{3}^{k}}}=\frac{2 k+1}{2 h}\left[Q_{3}^{+}-(-1)^{k} Q_{3}^{-}\right]-\frac{1}{A_{1} A_{2}} \underline{Q_{3}^{k}}+\left(k_{1}+k_{2}\right) Q_{3}^{k} .
$$

Now instead of the finite 3-D system of the differential equations in displacements (14) we have an infinite system of 2-D differential equations for coefficients of the Legendre's polynomial series expansion. In order to simplify the problem approximate theory has to be developed, and only the finite number of members has to be taken into account in the expansion (20) and in all the previous relations. For example, if we consider $n$-order approximate shell theory, only $n+1$ members in the expansion (20) are taken into account:

$$
\begin{aligned}
& u_{i}(\mathbf{x}, t)=\sum_{k=0}^{n} u_{i}^{k}\left(\mathbf{x}_{\alpha}, t\right) P_{k}(\omega), \\
& \sigma_{i j}(\mathbf{x}, t)=\sum_{k=0}^{n} \sigma_{i j}^{k}\left(\mathbf{x}_{\alpha}, t\right) P_{k}(\omega), \\
& \varepsilon_{i j}(\mathbf{x}, t)=\sum_{k=0}^{n} \varepsilon_{i j}^{k}\left(\mathbf{x}_{\alpha}, t\right) P_{k}(\omega), \\
& \theta(\mathbf{x}, t)=\sum_{k=0}^{n} \theta^{k}\left(\mathbf{x}_{\alpha}, t\right) P_{k}(\omega) .
\end{aligned}
$$

In this case we consider that $u_{i}^{k}=0, \sigma_{i j}^{k}=0, \varepsilon_{i j}^{k}=0$ and $\theta^{k}=0$ for $k<0$ and for $k>n$.

Order of the system of differential equations depends on assumption regarding thickness distribution of the stressstrain parameters of the shell.

\section{Vekua's Shell Equations}

In the case if only the first two terms of the Legendre polynomials series are considered in the expansion (20) we have the first approximation shell theory which is usually referred to as Vekua's shell theory. In this case the thermodynamic parameters, which describe the state of the shell, can be presented in the form

$$
\begin{gathered}
\sigma_{i j}(\mathbf{x}, t)=\sigma_{i j}^{0}\left(\mathbf{x}_{\alpha}, t\right) P_{0}(\omega)+\sigma_{i j}^{1}\left(\mathbf{x}_{\alpha}, t\right) P_{1}(\omega), \\
u_{i}(\mathbf{x}, t)=u_{i}^{0}\left(\mathbf{x}_{\alpha}, t\right) P_{0}(\omega)+u_{i}^{1}\left(\mathbf{x}_{v}, t\right) P_{1}(\omega),
\end{gathered}
$$

$$
\begin{gathered}
\varepsilon_{i j}(\mathbf{x}, t)=\varepsilon_{i j}^{0}\left(\mathbf{x}_{\alpha}, t\right) P_{0}(\omega)+\varepsilon_{i j}^{1}\left(\mathbf{x}_{\alpha}, t\right) P_{1}(\omega), \\
\theta(\mathbf{x}, t)=\theta^{0}\left(\mathbf{x}_{\alpha}, t\right) P_{0}(\omega)+\theta^{1}\left(\mathbf{x}_{\alpha}, t\right) P_{1}(\omega),
\end{gathered}
$$

where coefficients of the expansion are

$$
\begin{gathered}
u_{i}^{0}\left(\mathbf{x}_{\alpha}, t\right)=\frac{1}{2 h} \int_{-h}^{h} u_{i}\left(\mathbf{x}_{\alpha}, x_{3}, t\right) d x_{3}, \\
u_{i}^{1}\left(\mathbf{x}_{\alpha}, t\right)=\frac{3}{2 h} \int_{-h}^{h} u_{i}\left(\mathbf{x}_{\alpha}, x_{3}, t\right) x_{3} d x_{3}, \\
\varepsilon_{i j}^{0}\left(\mathbf{x}_{\alpha}, t\right)=\frac{1}{2 h} \int_{-h}^{h} \varepsilon_{i j}\left(\mathbf{x}_{\alpha}, x_{3}, t\right) d x_{3}, \\
\varepsilon_{i j}^{1}\left(\mathbf{x}_{\alpha}, t\right)=\frac{3}{2 h} \int_{-h}^{h} \varepsilon_{i j}\left(\mathbf{x}_{\alpha}, x_{3}, t\right) x_{3} d x_{3}, \\
\sigma_{i j}^{0}\left(\mathbf{x}_{\alpha}, t\right)=\frac{1}{2 h} \int_{-h}^{h} \sigma_{i j}\left(\mathbf{x}_{\alpha}, x_{3}, t\right) d x_{3}, \\
\sigma_{i j}^{1}\left(\mathbf{x}_{\alpha}, t\right)=\frac{3}{2 h} \int_{-h}^{h} \sigma_{i j}\left(\mathbf{x}_{\alpha}, x_{3}, t\right) x_{3} d x_{3}, \\
\theta^{0}\left(\mathbf{x}_{\alpha}, t\right)=\frac{1}{2 h} \int_{-h}^{h} \theta\left(\mathbf{x}_{\alpha}, x_{3}, t\right) d x_{3}, \\
\theta^{1}\left(\mathbf{x}_{\alpha}, t\right)=\frac{3}{2 h} \int_{-h}^{h} \theta\left(\mathbf{x}_{\alpha}, x_{3}, t\right) x_{3} d x_{3} .
\end{gathered}
$$

The equations of motion (26) in this case have the form

$$
\begin{aligned}
& \frac{\partial\left(A_{2} \sigma_{11}^{0}\right)}{\partial x_{1}}+\frac{\partial\left(A_{1} \sigma_{12}^{0}\right)}{\partial x_{2}}+\sigma_{12}^{0} \frac{\partial A_{1}}{\partial x_{2}}+\sigma_{13}^{0} A_{1} A_{2} k_{1}-\sigma_{22}^{0} \frac{\partial A_{2}}{\partial x_{1}} \\
& -\underline{\sigma_{13}^{0}}+A_{1} A_{2} f_{1}^{0}=\rho A_{1} A_{2} \ddot{u}_{1}^{0}, \\
& \frac{\partial\left(A_{2} \sigma_{21}^{0}\right)}{\partial x_{1}}+\frac{\partial\left(A_{1} \sigma_{22}^{0}\right)}{\partial x_{2}}+\sigma_{12}^{0} \frac{\partial A_{2}}{\partial x_{1}}+\sigma_{23}^{0} A_{1} A_{2} k_{2}-\sigma_{11}^{0} \frac{\partial A_{2}}{\partial x_{1}} \\
& -\underline{\sigma_{23}^{0}}+A_{1} A_{2} f_{2}^{0}=\rho A_{1} A_{2} \ddot{u}_{2}^{0}, \\
& \frac{\partial\left(A_{2} \sigma_{31}^{0}\right)}{\partial x_{1}}+\frac{\partial\left(A_{1} \sigma_{32}^{0}\right)}{\partial x_{2}}-\sigma_{11}^{0} A_{1} A_{2} k_{1}-\sigma_{22}^{0} A_{1} A_{2} k_{2} \\
& -\underline{\sigma_{33}^{0}}+A_{1} A_{2} f_{3}^{0}=\rho A_{1} A_{2} \ddot{u}_{3}^{0}, \\
& \frac{\partial\left(A_{2} \sigma_{11}^{1}\right)}{\partial x_{1}}+\frac{\partial\left(A_{1} \sigma_{12}^{1}\right)}{\partial x_{2}}+\sigma_{12}^{1} \frac{\partial A_{1}}{\partial x_{2}}+\sigma_{13}^{1} A_{1} A_{2} k_{1}-\sigma_{22}^{1} \frac{\partial A_{2}}{\partial x_{1}} \\
& -\frac{\sigma_{13}^{1}}{\sigma_{23}}+A_{1} A_{2} f_{1}^{1}=\rho A_{1} A_{2} \ddot{u}_{1}^{1}, \\
& \frac{\partial\left(A_{2} \sigma_{21}^{1}\right)}{\partial x_{1}}+\frac{\partial\left(A_{1} \sigma_{22}^{1}\right)}{\partial x_{2}}+\sigma_{12}^{1} \frac{\partial A_{2}}{\partial x_{1}}+\sigma_{23}^{1} A_{1} A_{2} k_{2}-\sigma_{11}^{1} \frac{\partial A_{2}}{\partial x_{1}}
\end{aligned}
$$




$$
\begin{gathered}
\frac{\partial\left(A_{2} \sigma_{31}^{1}\right)}{\partial x_{1}}+\frac{\partial\left(A_{1} \sigma_{32}^{1}\right)}{\partial x_{2}}-\sigma_{11}^{1} A_{1} A_{2} k_{1}-\sigma_{22}^{1} A_{1} A_{2} k_{2} \\
\quad-\underline{\sigma_{33}^{1}}+A_{1} A_{2} f_{3}^{1}=\rho A_{1} A_{2} \ddot{u}_{3}^{1} .
\end{gathered}
$$

The kinematic Cauchy relations (28) have the form

$$
\begin{aligned}
& \varepsilon_{11}^{0}=\frac{1}{A_{1}} \frac{\partial u_{1}^{0}}{\partial x_{1}}+\frac{1}{A_{1} A_{2}} \frac{\partial A_{1}}{\partial x_{2}} u_{2}^{0}+k_{1} u_{3}^{0}, \\
& \varepsilon_{22}^{0}=\frac{1}{A_{2}} \frac{\partial u_{2}^{0}}{\partial x_{2}}+\frac{1}{A_{1} A_{2}} \frac{\partial A_{2}}{\partial x_{1}} u_{1}^{0}+k_{2} u_{3}^{0}, \\
& \varepsilon_{12}^{0}=\frac{1}{A_{2}}\left(\frac{\partial u_{1}^{0}}{\partial x_{2}}-\frac{1}{A_{1}} \frac{\partial A_{2}}{\partial x_{1}} u_{2}^{0}\right)+\frac{1}{A_{1}}\left(\frac{\partial u_{2}^{0}}{\partial x_{1}}-\frac{1}{A_{2}} \frac{\partial A_{1}}{\partial x_{2}} u_{1}^{0}\right) \text {, } \\
& \varepsilon_{13}^{0}=\frac{1}{A_{1}} \frac{\partial u_{3}^{0}}{\partial x_{1}}-k_{1} u_{1}^{0}+\frac{1}{h} u_{1}^{1} \\
& \varepsilon_{23}^{0}=\frac{1}{A_{2}} \frac{\partial u_{3}^{0}}{\partial x_{2}}-k_{2} u_{2}^{0}+\frac{1}{h} u_{2}^{1}, \quad \varepsilon_{33}^{0}=\frac{1}{h} u_{3}^{1} \text {, } \\
& \varepsilon_{11}^{1}=\frac{1}{A_{1}} \frac{\partial u_{1}^{1}}{\partial x_{1}}+\frac{1}{A_{1} A_{2}} \frac{\partial A_{1}}{\partial x_{2}} u_{2}^{1}+k_{1} u_{3}^{1}, \\
& \varepsilon_{22}^{1}=\frac{1}{A_{2}} \frac{\partial u_{2}^{1}}{\partial x_{2}}+\frac{1}{A_{1} A_{2}} \frac{\partial A_{2}}{\partial x_{1}} u_{1}^{1}+k_{2} u_{3}^{1}, \\
& \varepsilon_{12}^{1}=\frac{1}{A_{2}}\left(\frac{\partial u_{1}^{1}}{\partial x_{2}}-\frac{1}{A_{1}} \frac{\partial A_{2}}{\partial x_{1}} u_{2}^{1}\right)+\frac{1}{A_{1}}\left(\frac{\partial u_{2}^{1}}{\partial x_{1}}-\frac{1}{A_{2}} \frac{\partial A_{1}}{\partial x_{2}} u_{1}^{1}\right) \\
& \varepsilon_{13}^{1}=\frac{1}{A_{1}} \frac{\partial u_{3}^{1}}{\partial x_{1}}-k_{1} u_{1}^{1} \\
& \varepsilon_{23}^{1}=\frac{1}{A_{2}} \frac{\partial u_{3}^{1}}{\partial x_{2}}-k_{2} u_{2}^{1}, \quad \varepsilon_{33}^{1}=0 \text {. }
\end{aligned}
$$

The generalized Hooke's law for homogeneous isotropic material (30) has the form

$$
\begin{gathered}
\sigma_{11}^{0}=(\lambda+2 \mu) \varepsilon_{11}^{0}+\lambda\left(\varepsilon_{22}^{0}+\varepsilon_{33}^{0}\right)+\beta_{T} \theta^{0}, \\
\sigma_{22}^{0}=(\lambda+2 \mu) \varepsilon_{22}^{0}+\lambda\left(\varepsilon_{11}^{0}+\varepsilon_{33}^{0}\right)+\beta_{T} \theta^{0}, \\
\sigma_{33}^{0}=(\lambda+2 \mu) \varepsilon_{22}^{0}+\lambda\left(\varepsilon_{11}^{0}+\varepsilon_{22}^{0}\right)+\beta_{T} \theta^{0}, \\
\sigma_{12}^{0}=\mu \varepsilon_{12}^{0}, \quad \sigma_{13}^{0}=\mu \varepsilon_{13}^{0}, \quad \sigma_{23}^{0}=\mu \varepsilon_{23}^{0}, \\
\sigma_{11}^{1}=(\lambda+2 \mu) \varepsilon_{11}^{1}+\lambda\left(\varepsilon_{22}^{1}+\varepsilon_{33}^{1}\right)+\beta_{T} \theta^{1}, \\
\sigma_{22}^{1}=(\lambda+2 \mu) \varepsilon_{22}^{1}+\lambda\left(\varepsilon_{11}^{1}+\varepsilon_{33}^{1}\right)+\beta_{T} \theta^{1}, \\
\sigma_{33}^{1}=(\lambda+2 \mu) \varepsilon_{22}^{1}+\lambda\left(\varepsilon_{11}^{1}+\varepsilon_{22}^{1}\right)+\beta_{T} \theta^{1}, \\
\sigma_{12}^{1}=\mu \varepsilon_{12}^{1}, \quad \sigma_{13}^{1}=\mu \varepsilon_{13}^{1}, \quad \sigma_{23}^{1}=\mu \varepsilon_{23}^{1} .
\end{gathered}
$$

Substitution of the kinematic Cauchy relations (39) with the generalized Hooke's law (40) and the result of the equations of motion (38) give us the 2-D equations in displacements for Vekua's shell theory in the form

$$
\begin{aligned}
& L_{i j}^{00} u_{j}^{0}+L_{i j}^{01} u_{j}^{1}+L_{i}^{0} \theta^{0}+A_{1} A_{2} b_{i}^{k}=\rho A_{1} A_{2} \ddot{u}_{i}^{k}, \\
& L_{i j}^{10} u_{j}^{0}+L_{i j}^{11} u_{j}^{1}+L_{i}^{1} \theta^{1}+A_{1} A_{2} b_{i}^{k}=\rho A_{1} A_{2} \ddot{u}_{i}^{k},
\end{aligned}
$$

and the equation of heat conductivity in the form

$$
\begin{aligned}
& \lambda_{T} \Delta \theta^{0}+\underline{\underline{Q_{3}^{0}}}+\chi^{0}=c_{\varepsilon} \dot{\theta}^{0}+\beta_{\tau} \theta_{0}\left(\nabla \cdot \dot{\mathbf{u}}^{0}+\frac{1}{h} \dot{u}_{3}^{1}\right), \\
& \lambda_{T} \Delta \theta^{1}+\underline{\underline{Q_{3}^{1}}}+\chi^{1}=c_{\varepsilon} \dot{\theta}^{1}+\beta_{\tau} \theta_{0} \nabla \cdot \dot{\mathbf{u}}^{1},
\end{aligned}
$$

where $Q_{3}^{+}, Q_{3}^{-}, Q_{3}^{0}$, and $Q_{3}^{1}$ depend on thermal conditions on the outer sides $\Omega^{+}$and $\Omega^{-}$of the shell. In the case if temperatures $\theta^{+}$and $\theta^{-}$are prescribed on $\Omega^{+}$and $\Omega^{-}$, respectively, they have the form

$$
\begin{aligned}
& Q_{3}^{+}-Q_{3}^{-}=\frac{3}{4 h}\left(\theta^{+}+\theta^{-}\right)+\frac{3 \theta^{0}}{2 h}, \quad Q_{3}^{0}=\frac{1}{2 h}\left(\theta^{+}-\theta^{-}\right), \\
& Q_{3}^{+}+Q_{3}^{-}=\frac{3}{2 h}\left(\theta^{+}-\theta^{-}\right)-\frac{5 \theta^{1}}{2 h}, \quad Q_{3}^{1}=\frac{3}{2 h}\left(\theta^{+}+\theta^{-}\right)-\frac{3 \theta^{1}}{2 h} .
\end{aligned}
$$

Differential operators that appear in the equations of thermoelasticity (41) and heat conductivity (42) for shells of arbitrary geometry are presented in the Appendix Section.

\section{Timoshenko's Shell Equations}

Timoshenko's theory of shells is based on assumptions concerning the value and distribution of the stress-strain state of the shell. Thus, according to static assumptions $\sigma_{33}=0$ and according to kinematic assumptions $\varepsilon_{33}=0$. In this theory the thermodynamic state of shells is determined by quantities specified on the middle surface. The stress state is characterized by the normal $n_{\alpha \alpha}$, tangential $n_{\alpha \beta}(\alpha \neq \beta)$, and shear $n_{\alpha 3}$ forces, as well as the bending $m_{\alpha \alpha}$ and twisting $m_{\alpha \beta}(\alpha \neq \beta)$ moments. They are defined as follows:

$$
\begin{gathered}
n_{\alpha \beta}=\int_{-h}^{h} \sigma_{\alpha \beta} d x_{3}, \quad n_{3 \beta}=\int_{-h}^{h} \sigma_{3 \beta} d x_{3}, \\
m_{\alpha \beta}=\int_{-h}^{h} \sigma_{\alpha \beta} x_{3} d x_{3} .
\end{gathered}
$$

Comparison with (37) gives us the following relation between corresponding parameters in Vekua's and Timoshenko's theories:

$$
\sigma_{i \beta}^{0} \sim \frac{n_{i \beta}}{2 h}, \quad \sigma_{\alpha \beta}^{1} \sim \frac{3 m_{\alpha \beta}}{2 h^{2}} .
$$

Components $\sigma_{33}^{0}$ and $\sigma_{33}^{1}$ are not taken into account in Timoshenko's theory of shells. That follows also from the static hypothesis.

Displacements in the Timoshenko's theory of shells are defined by vectors $\mathbf{u}\left(\mathbf{x}_{\alpha}, t\right)$ and $\gamma\left(\mathbf{x}_{\alpha}, t\right)$ with components $u_{i}$, 
$i=1,2,3$ and $\gamma_{\alpha}, \alpha=1,2$, respectively. They correspond to displacements of the middle surface and rotation of the elements perpendicular to the middle surface in the planes $\left(x_{\alpha}, x_{3}\right)$. These parameters are related to the coefficients of the displacements expansion in the Vekua's theory in the following way:

$$
u_{i}^{0} \sim u_{i}, \quad u_{\alpha}^{1} \sim \gamma_{\alpha} h
$$

Component $u_{3}^{1}$ is not taken into account in Timoshenko's theory of shells.

Deformations in Timoshenko's theory are determined by the relations

$$
\varepsilon_{\alpha \beta}=e_{\alpha \beta}+\kappa_{\alpha \beta} x_{3}, \quad \varepsilon_{\alpha 3}=e_{\alpha 3} .
$$

Roughly speaking components $e_{\alpha \beta}$ correspond to the tensioncompression deformation of the middle surface, components $e_{\alpha 3}$ to the transversal shear deformation, and components $\kappa_{\alpha \beta}$ to the bending and twisting middle surface, respectively. The following formulas give us relations with corresponding quantities in Vekua's theory:

$$
\varepsilon_{\alpha i}^{0} \sim e_{\alpha i}, \quad \varepsilon_{\alpha \beta}^{1} \sim \kappa_{\alpha \beta} .
$$

Component $\varepsilon_{33}^{0}$ and $\varepsilon_{33}^{1}$ are not taken into account in the Timoshenko's theory of shells. That follows also from the kinematic hypothesis.

The kinematic Cauchy relations in Timoshenko's theory of shells have the following form:

$$
\begin{aligned}
& e_{11}=\frac{1}{A_{1}} \frac{\partial u_{1}}{\partial x_{1}}+\frac{1}{A_{1} A_{2}} \frac{\partial A_{1}}{\partial x_{2}} u_{2}+k_{1} u_{3} \\
& e_{22}=\frac{1}{A_{2}} \frac{\partial u_{2}}{\partial x_{2}}+\frac{1}{A_{1} A_{2}} \frac{\partial A_{2}}{\partial x_{1}} u_{1}+k_{2} u_{3} \text {, } \\
& e_{12}=\frac{1}{A_{2}}\left(\frac{\partial u_{1}}{\partial x_{2}}-\frac{1}{A_{1}} \frac{\partial A_{2}}{\partial x_{1}} u_{2}\right)+\frac{1}{A_{1}}\left(\frac{\partial u_{2}}{\partial x_{1}}-\frac{1}{A_{2}} \frac{\partial A_{1}}{\partial x_{2}} u_{1}\right) \text {, } \\
& e_{13}=\frac{1}{A_{1}} \frac{\partial u_{3}}{\partial x_{1}}-k_{1} u_{1}+\frac{1}{h} \gamma_{1}, \\
& \varepsilon_{23}^{0}=\frac{1}{A_{2}} \frac{\partial u_{3}}{\partial x_{2}}-k_{2} u_{2}+\frac{1}{h} \gamma_{2}, \\
& \varepsilon_{33}^{0}=0 \text {, } \\
& \kappa_{11}=\frac{1}{A_{1}} \frac{\partial \gamma_{1}}{\partial x_{1}}+\frac{1}{A_{1} A_{2}} \frac{\partial A_{1}}{\partial x_{2}} \gamma_{2}, \\
& \kappa_{22}=\frac{1}{A_{2}} \frac{\partial \gamma_{2}}{\partial x_{2}}+\frac{1}{A_{1} A_{2}} \frac{\partial A_{2}}{\partial x_{1}} \gamma_{1} \text {, } \\
& \kappa_{12}=\frac{1}{A_{1}} \frac{\partial \gamma_{2}}{\partial x_{1}}-\frac{1}{A_{1} A_{2}} \frac{\partial A_{1}}{\partial x_{2}} \gamma_{1}+k_{1} \omega_{2} \text {, } \\
& \kappa_{21}=\frac{1}{A_{2}} \frac{\partial \gamma_{1}}{\partial x_{2}}-\frac{1}{A_{1} A_{2}} \frac{\partial A_{2}}{\partial x_{1}} \gamma_{2}+k_{2} \omega_{1} \text {, }
\end{aligned}
$$

$\omega_{1}=\frac{1}{A_{1}} \frac{\partial u_{2}}{\partial x_{1}}-\frac{1}{A_{1} A_{2}} \frac{\partial A_{1}}{\partial x_{2}} u_{1}$,
$\omega_{2}=\frac{1}{A_{2}} \frac{\partial u_{1}}{\partial x_{2}}-\frac{1}{A_{1} A_{2}} \frac{\partial A_{2}}{\partial x_{1}} u_{2}$.

The equations of motion have the form

$$
\begin{aligned}
& \frac{\partial\left(A_{2} n_{11}\right)}{\partial x_{1}}+\frac{\partial\left(A_{1} n_{12}\right)}{\partial x_{2}}+n_{12} \frac{\partial A_{1}}{\partial x_{2}}-n_{22} \frac{\partial A_{2}}{\partial x_{1}}+n_{13} A_{1} A_{2} k_{1} \\
& +A_{1} A_{2} f_{1}^{k}=\rho A_{1} A_{2} \ddot{u}_{1} \text {, } \\
& \frac{\partial\left(A_{2} n_{12}\right)}{\partial x_{1}}+\frac{\partial\left(A_{1} n_{22}\right)}{\partial x_{2}}+n_{21} \frac{\partial A_{2}}{\partial x_{1}}-n_{11} \frac{\partial A_{2}}{\partial x_{1}}+n_{23} A_{1} A_{2} k_{2} \\
& +A_{1} A_{2} f_{2}^{k}=\rho A_{1} A_{2} \ddot{u}_{2}, \\
& \frac{\partial\left(A_{2} n_{31}\right)}{\partial x_{1}}+\frac{\partial\left(A_{1} n_{32}\right)}{\partial x_{2}}-n_{11} A_{1} A_{2} k_{1}-n_{22} A_{1} A_{2} k_{2} \\
& +A_{1} A_{2} f_{3}^{k}=\rho A_{1} A_{2} \ddot{u}_{3} \text {, } \\
& \frac{\partial\left(A_{2} m_{11}\right)}{\partial x_{1}}+\frac{\partial\left(A_{1} m_{12}\right)}{\partial x_{2}}+m_{12} \frac{\partial A_{1}}{\partial x_{2}}-m_{22} \frac{\partial A_{2}}{\partial x_{1}} \\
& \text { - } n_{13} A_{1} A_{2}=\rho A_{1} A_{2} h \ddot{\gamma}_{1} \text {, } \\
& \frac{\partial\left(A_{2} m_{21}\right)}{\partial x_{1}}+\frac{\partial\left(A_{1} m_{22}\right)}{\partial x_{2}}+m_{21} \frac{\partial A_{2}}{\partial x_{1}}-m_{11} \frac{\partial A_{2}}{\partial x_{1}} \\
& \text { - } n_{23} A_{1} A_{2}=\rho A_{1} A_{2} h \ddot{\gamma}_{2} \text {, } \\
& n_{12}+m_{12} k_{1}=n_{21}+m_{21} k_{2} \text {. }
\end{aligned}
$$

The generalized Hooke's law for Timoshenko's theory of shells has the form

$$
\begin{gathered}
n_{\alpha \alpha}=\frac{2 E h}{1-v^{2}}\left[e_{\alpha \alpha}+v e_{\beta \beta}-(1+v) \alpha_{T} \theta^{0}\right], \\
n_{12}=\frac{E h}{1+v} e_{12}, \quad n_{\alpha 3}=\frac{E h}{1+v} e_{\alpha 3}, \\
m_{\alpha \alpha}=\frac{2 E h}{1-v^{2}}\left[\kappa_{\alpha \alpha}+v \kappa_{\beta \beta}-(1+v) \alpha_{T} \theta^{1}\right] . \\
m_{12}=\frac{2 E h^{3}}{3(1+v)} e_{12} .
\end{gathered}
$$

Such form of the generalized Hooke's law follows from the static hypothesis according to which $\sigma_{33}=0$, and therefore

$$
\varepsilon_{33}=-\frac{v}{1-v}\left(\varepsilon_{11}+\varepsilon_{22}\right)
$$

Substituting kinematic relations (49) with generalized Hooke's law (51) and considering the result of the equations of 
motion (50) we obtain the following system of the differential equations in the form of displacements:

$$
\begin{gathered}
L_{i j}^{00} u_{j}+L_{i \beta}^{01} \gamma_{\beta}+L_{i}^{0} \theta^{0}+A_{1} A_{2} b_{i}=\rho A_{1} A_{2} \ddot{u}_{i}, \\
L_{\alpha j}^{10} u_{j}+L_{\alpha \beta}^{11} \gamma_{\beta}+L_{\alpha}^{1} \theta^{1}+A_{1} A_{2} m_{\alpha}=\rho A_{1} A_{2} h \ddot{\gamma}_{\alpha} .
\end{gathered}
$$

Differential operators that appear in (53) for shells of arbitrary geometry are presented in the Appendix Section.

The equations of heat conductivity in Timoshenko's theory of shells have the same form as in the Vekua's shells theory, that is, defined by (42).

Components of the stress tensor can be calculated from the equations

$$
\begin{gathered}
\sigma_{\alpha \beta}(\mathbf{x})=\frac{n_{\alpha \beta}\left(\mathbf{x}_{\alpha}\right)}{2 h}+\frac{3 m_{\alpha \beta}\left(\mathbf{x}_{\alpha}\right) x_{3}}{h^{3}}, \\
\sigma_{\alpha 3}(\mathbf{x})=\frac{n_{\alpha 3}\left(\mathbf{x}_{\alpha}\right)}{2 h}, \quad \sigma_{33}(\mathbf{x})=0 .
\end{gathered}
$$

The equations presented here allow us calculate stressstrain state of the shells under static and dynamic action of mechanical and thermal load.

\section{Kirchhoff-Love's Shell Equations}

In the classical Kirchhoff-Love's theory of shells in addition to the assumptions of Timoshenko's theory it is assumed that $\varepsilon_{\alpha 3}=0$ and that the angles of rotation of the normal-tomiddle surface vector become dependent; they are given by the equations

$$
u_{\alpha}^{1}=-\frac{1}{A_{\alpha}} \frac{\partial u_{3}^{0}}{\partial x_{\alpha}}+k_{\alpha} u_{\alpha}^{0}, \quad \text { or } \gamma_{\alpha}=-\frac{1}{A_{\alpha}} \frac{\partial u_{3}}{\partial x_{\alpha}}+k_{\alpha} u_{\alpha} .
$$

Substituting (55) with the kinematic equations for Timoshenko's shell theory we obtain kinematic equations for Kirchhoff-Love's shell theory in the form

$$
\begin{gathered}
e_{11}=\frac{1}{A_{1}} \frac{\partial u_{1}}{\partial x_{1}}+\frac{1}{A_{1} A_{2}} \frac{\partial A_{1}}{\partial x_{2}} u_{2}+k_{1} u_{3}, \\
e_{22}=\frac{1}{A_{2}} \frac{\partial u_{2}}{\partial x_{2}}+\frac{1}{A_{1} A_{2}} \frac{\partial A_{2}}{\partial x_{1}} u_{1}+k_{2} u_{3}, \\
e_{12}=\frac{1}{A_{2}}\left(\frac{\partial u_{1}}{\partial x_{2}}-\frac{1}{A_{1}} \frac{\partial A_{2}}{\partial x_{1}} u_{2}\right)+\frac{1}{A_{1}}\left(\frac{\partial u_{2}}{\partial x_{1}}-\frac{1}{A_{2}} \frac{\partial A_{1}}{\partial x_{2}} u_{1}\right), \\
\kappa_{11}=\frac{1}{A_{1}} \frac{\partial \gamma_{1}}{\partial x_{1}}-\frac{1}{A_{1} A_{2}} \frac{\partial A_{1}}{\partial x_{2}}\left(\frac{1}{A_{2}} \frac{\partial u_{3}}{\partial x_{2}}+k_{2} u_{2}\right), \\
\kappa_{22}=\frac{1}{A_{2}} \frac{\partial \gamma_{2}}{\partial x_{2}}-\frac{1}{A_{1} A_{2}} \frac{\partial A_{2}}{\partial x_{1}}\left(\frac{1}{A_{1}} \frac{\partial u_{3}}{\partial x_{1}}-k_{1} u_{1}\right), \\
\kappa_{12}=\frac{1}{A_{1} A_{2}} \frac{\partial A_{1}}{\partial x_{2}}\left(\frac{1}{A_{1}} \frac{\partial u_{3}}{\partial x_{1}}-k_{1} u_{1}\right) \\
-\frac{1}{A_{1}} \frac{\partial \gamma_{2}}{\partial x_{1}}\left(\frac{1}{A_{2}} \frac{\partial u_{3}}{\partial x_{2}}-k_{2} u_{2}\right)+k_{1} \omega_{2},
\end{gathered}
$$

$$
\begin{aligned}
\kappa_{21}= & \frac{1}{A_{1} A_{2}} \frac{\partial A_{2}}{\partial x_{1}}\left(\frac{1}{A_{2}} \frac{\partial u_{3}}{\partial x_{2}}-k_{2} u_{2}\right) \\
& -\frac{1}{A_{2}} \frac{\partial}{\partial x_{2}}\left(\frac{1}{A_{1}} \frac{\partial u_{3}}{\partial x_{1}}-k_{1} u_{1}\right)+k_{2} \omega_{1}, \\
\omega_{1}= & \frac{1}{A_{1}} \frac{\partial u_{2}}{\partial x_{1}}-\frac{1}{A_{1} A_{2}} \frac{\partial A_{1}}{\partial x_{2}} u_{1}, \\
\omega_{2}= & \frac{1}{A_{2}} \frac{\partial u_{1}}{\partial x_{2}}-\frac{1}{A_{1} A_{2}} \frac{\partial A_{2}}{\partial x_{1}} u_{2} .
\end{aligned}
$$

From two last equations of motion for Timoshenko's shell theory (50) we can find

$n_{13}=\frac{1}{A_{1} A_{2}}\left[\frac{\partial\left(A_{2} m_{11}\right)}{\partial x_{1}}+\frac{\partial\left(A_{1} m_{21}\right)}{\partial x_{2}}+m_{12} \frac{\partial A_{1}}{\partial x_{2}}-m_{22} \frac{\partial A_{2}}{\partial x_{1}}\right]$,
$n_{23}=\frac{1}{A_{1} A_{2}}\left[\frac{\partial\left(A_{2} m_{12}\right)}{\partial x_{1}}+\frac{\partial\left(A_{1} m_{22}\right)}{\partial x_{2}}+m_{21} \frac{\partial A_{2}}{\partial x_{1}}-m_{11} \frac{\partial A_{1}}{\partial x_{2}}\right]$.

Substituting them with other equations of motion (50) we obtain

$$
\begin{aligned}
& \frac{\partial\left(A_{2} n_{11}\right)}{\partial x_{1}}+\frac{\partial\left(A_{1} n_{12}\right)}{\partial x_{2}}+n_{12} \frac{\partial A_{1}}{\partial x_{2}}-n_{22} \frac{\partial A_{2}}{\partial x_{1}} \\
& +k_{1}\left[\frac{\partial\left(A_{2} m_{11}\right)}{\partial x_{1}}+\frac{\partial\left(A_{1} m_{21}\right)}{\partial x_{2}}\right. \\
& \left.+m_{12} \frac{\partial A_{1}}{\partial x_{2}}-m_{22} \frac{\partial A_{2}}{\partial x_{1}}\right] \\
& +A_{1} A_{2} f_{1}^{k}=\rho A_{1} A_{2} \ddot{u}_{1} \text {, } \\
& \frac{\partial\left(A_{2} n_{12}\right)}{\partial x_{1}}+\frac{\partial\left(A_{1} n_{22}\right)}{\partial x_{2}}+n_{21} \frac{\partial A_{2}}{\partial x_{1}}-n_{11} \frac{\partial A_{2}}{\partial x_{1}} \\
& +k_{2}\left[\frac{\partial\left(A_{2} m_{12}\right)}{\partial x_{1}}+\frac{\partial\left(A_{1} m_{22}\right)}{\partial x_{2}}\right. \\
& \left.+m_{21} \frac{\partial A_{2}}{\partial x_{1}}-m_{11} \frac{\partial A_{1}}{\partial x_{2}}\right] \\
& +A_{1} A_{2} f_{2}^{k}=\rho A_{1} A_{2} \ddot{u}_{2} \text {, } \\
& \frac{\partial}{\partial x_{1}}\left\{\frac { 1 } { A _ { 1 } } \left[\frac{\partial\left(A_{2} m_{11}\right)}{\partial x_{1}}+\frac{\partial\left(A_{1} m_{21}\right)}{\partial x_{2}}\right.\right. \\
& \left.\left.+m_{12} \frac{\partial A_{1}}{\partial x_{2}}-m_{22} \frac{\partial A_{2}}{\partial x_{1}}\right]\right\}
\end{aligned}
$$




$$
\begin{gathered}
+\frac{\partial}{\partial x_{2}}\left\{\frac { 1 } { A _ { 2 } } \left[\frac{\partial\left(A_{2} m_{12}\right)}{\partial x_{1}}+\frac{\partial\left(A_{1} m_{22}\right)}{\partial x_{2}}\right.\right. \\
\left.\left.+m_{21} \frac{\partial A_{2}}{\partial x_{1}}-m_{11} \frac{\partial A_{1}}{\partial x_{2}}\right]\right\} \\
-n_{11} A_{1} A_{2} k_{1}-n_{22} A_{1} A_{2} k_{2}+A_{1} A_{2} f_{3}^{k}=\rho A_{1} A_{2} \ddot{u}_{3} .
\end{gathered}
$$

In the same way substituting kinematic relations (56) with generalized Hooke's law (51) and considering the result of the equations of motion (58) we obtain the following system of the differential equations in the form of displacements for Kirchhoff-Love's shell theory:

$$
L_{i j} u_{j}+\sum_{k=0}^{1} L_{i}^{k} \theta^{k}+A_{1} A_{2} b_{i}=\rho A_{1} A_{2} \ddot{u}_{i}
$$

Differential operators that appear in (59) for shells of arbitrary geometry have more complicated form; therefore they were not presented here.

The equations of heat conductivity in Kirchhoff-Love's theory of shells have the same form as in Vekua's shells theory, that is, defined by (42).

Components of the stress tensor can be calculated in the same way as for Timoshenko's shell theory using (54).

\section{Comparative Study and Applications}

Developed here is high order shell theory that gives us the full system of the equations for studying thermodynamical state of the thin-walled structures with high accuracy. We can keep in the polynomial expansion (20) as many terms as it is necessary for approximation of the stress-strain state with necessary accuracy. The only problem that may occur is that, on one hand more members one keeps, the more accurate results he has on the other hand the more members one keeps, the more complicated the system of equations he has to solve. Therefore general suggestion is that one has to keep as many members as it is necessary for getting results with necessary accuracy, but not more. We consider here in more detail first order theory which takes into account two members in the expansion (20) that theory is usually referred to as Vekua's shell theory. Let us compare Vekua's shell theory with classical Timoshenko's and Kirchhoff-Love's shell theories.

Comparison of Vekua's, Timoshenko's, and KirchhoffLove's shell theories has been done in Table 1.

From Table 1 it follows that Vekua's shell theory considers all components of the stress-strain state in linear approximation. Classical theories are based on physical assumptions regarding stress and strain distribution. For example, according to static hypothesis $\sigma_{33}=0$ and according to kinematic hypothesis $\varepsilon_{33}=0$, and therefore components $\sigma_{33}^{0}, \sigma_{33}^{1}$, and $\varepsilon_{33}^{0}, \varepsilon_{33}^{1}$ have no analogy in Timoshenko's and Kirchhoff-Love's shell theories. But on the other hand in classical theories $\varepsilon_{33}$ is defined from the Hooke's law using (52). Also in Kirchhoff-Love's shell theory $\varepsilon_{\alpha 3}=0$ and in accordance with Hooke's law $\sigma_{\alpha 3}=0$, and therefore $n_{\alpha 3}=0$. But in fact $\sigma_{\alpha 3}$ and $n_{\alpha 3}$ are defined from the equations of equilibrium. Unfortunately classical theories contain some logical contradictions; nevertheless they are very popular and are frequently used in engineering applications.

Systems of differential equations in displacements for Vekua's (41), Timoshenko's (53), and Kirchhoff-Love's (59) theories for the case of arbitrary geometry shells are very complicated because of the structure of the differential operator presented in the Appendix Section. In order to simplify analysis of the structure of those equations, let us consider how they look for plates and cylindrical shells.

7.1. Equations in the Form of Displacements for Plates. All equations that specify thermodynamical state of the plates can be obtained from the general equations. In this case one has to substitute $A_{1}=A_{2}=1$ and $k_{1}=k_{2}=0$ and consider all the equations in Cartesian system of coordinates in order to obtain corresponding equations for the plates theory. We present in the following only differential equations in displacements for theories considered here.

For Vekua's theory differential equations in displacements have the form

$$
\begin{aligned}
& \mu \Delta u_{1}^{0}+(\lambda+\mu) \frac{\partial}{\partial x_{1}}\left(\frac{\partial u_{1}^{0}}{\partial x_{1}}+\frac{\partial u_{2}^{0}}{\partial x_{2}}\right)+\frac{\lambda}{h} \frac{\partial u_{3}^{1}}{\partial x_{1}} \\
& +\beta_{T} \frac{\partial \theta^{0}}{\partial x_{1}}+f_{1}^{0}=\rho \ddot{u}_{1}^{0}, \\
& \mu \Delta u_{2}^{0}+(\lambda+\mu) \frac{\partial}{\partial x_{2}}\left(\frac{\partial u_{1}^{0}}{\partial x_{1}}+\frac{\partial u_{2}^{0}}{\partial x_{2}}\right)+\frac{\lambda}{h} \frac{\partial u_{3}^{1}}{\partial x_{2}} \\
& +\beta_{T} \frac{\partial \theta^{0}}{\partial x_{2}}+f_{2}^{0}=\rho \ddot{u}_{2}^{0}, \\
& \mu \Delta u_{3}^{0}+\frac{\mu}{h}\left(\frac{\partial u_{1}^{1}}{\partial x_{1}}+\frac{\partial u_{2}^{1}}{\partial x_{2}}\right)+f_{3}^{0}=\rho \ddot{u}_{3}^{0} \text {, } \\
& \mu \Delta u_{1}^{1}+(\lambda+\mu) \frac{\partial}{\partial x_{1}}\left(\frac{\partial u_{1}^{1}}{\partial x_{1}}+\frac{\partial u_{2}^{1}}{\partial x_{2}}\right)-\frac{3 \mu}{h}\left(\frac{\partial u_{3}^{0}}{\partial x_{1}}+\frac{1}{h} u_{1}^{1}\right) \\
& +\beta_{T}\left(\frac{\partial \theta^{0}}{\partial x_{1}}+\frac{\partial \theta^{1}}{\partial x_{1}}\right)+f_{1}^{1}=\rho \ddot{u}_{1}^{1} \text {, } \\
& \mu \Delta u_{2}^{1}+(\lambda+\mu) \frac{\partial}{\partial x_{2}}\left(\frac{\partial u_{1}^{1}}{\partial x_{1}}+\frac{\partial u_{2}^{1}}{\partial x_{2}}\right)-\frac{3 \mu}{h}\left(\frac{\partial u_{3}^{0}}{\partial x_{2}}+\frac{1}{h} u_{2}^{1}\right) \\
& +\beta_{T}\left(\frac{\partial \theta^{0}}{\partial x_{2}}+\frac{\partial \theta^{1}}{\partial x_{2}}\right)+f_{2}^{1}=\rho \ddot{u}_{2}^{1}, \\
& \mu \Delta u_{3}^{1}-\frac{3 \lambda}{h}\left(\frac{\partial u_{1}^{0}}{\partial x_{1}}+\frac{\partial u_{2}^{0}}{\partial x_{2}}\right)+\frac{3(\lambda+2 \mu)}{h^{2}} u_{3}^{1}-\frac{3 \beta_{T}}{h} \theta^{0} \\
& +f_{3}^{1}=\rho \ddot{u}_{3}^{1} \text {. }
\end{aligned}
$$


TABLE 1

\begin{tabular}{|c|c|c|c|c|c|c|c|c|c|c|c|c|}
\hline Vekua & $\sigma_{11}^{0}$ & $\sigma_{12}^{0}$ & $\sigma_{13}^{0}$ & $\sigma_{22}^{0}$ & $\sigma_{23}^{0}$ & $\sigma_{33}^{0}$ & $\sigma_{11}^{1}$ & $\sigma_{12}^{1}$ & $\sigma_{13}^{1}$ & $\sigma_{22}^{1}$ & $\sigma_{23}^{1}$ & $\sigma_{33}^{1}$ \\
\hline Timoshenko & $n_{11}$ & $n_{12}$ & $n_{13}$ & $n_{22}$ & $n_{23}$ & & $m_{11}$ & $m_{12}$ & & $m_{22}$ & & \\
\hline Kirchhoff-Love & $n_{11}$ & $n_{12}$ & $n_{13}$ & $n_{22}$ & $n_{23}$ & & $m_{11}$ & $m_{12}$ & & $m_{22}$ & & \\
\hline Vekua & $\varepsilon_{11}^{0}$ & $\varepsilon_{12}^{0}$ & $\varepsilon_{13}^{0}$ & $\varepsilon_{22}^{0}$ & $\varepsilon_{23}^{0}$ & $\varepsilon_{33}^{0}$ & $\varepsilon_{11}^{1}$ & $\varepsilon_{12}^{1}$ & $\varepsilon_{13}^{1}$ & $\varepsilon_{22}^{1}$ & $\varepsilon_{23}^{1}$ & $\varepsilon_{33}^{1}$ \\
\hline Timoshenko & $e_{11}$ & $e_{12}$ & $e_{13}$ & $e_{22}$ & $e_{23}$ & & $\kappa_{11}$ & $\kappa_{12}$ & & $\kappa_{22}$ & & \\
\hline Kirchhoff-Love & $e_{11}$ & $e_{12}$ & & $e_{22}$ & & & $\kappa_{11}$ & $\kappa_{12}$ & & $\kappa_{22}$ & & \\
\hline Vekua & $u_{1}^{0}$ & $u_{2}^{0}$ & $u_{3}^{0}$ & & & & $u_{1}^{1}$ & $u_{2}^{1}$ & $u_{3}^{1}$ & & & \\
\hline Timoshenko & $u_{1}$ & $u_{2}$ & $u_{3}$ & & & & $\gamma_{1}$ & $\gamma_{2}$ & & & & \\
\hline Kirchhoff-Love & $u_{1}$ & $u_{2}$ & $u_{3}$ & & & & & & & & & \\
\hline
\end{tabular}

For Timoshenko's theory differential equations in displacements have the form

$$
\begin{aligned}
& \mu \Delta u_{1}+(\lambda+\mu) \frac{\partial}{\partial x_{1}}\left(\frac{\partial u_{1}}{\partial x_{1}}+\frac{\partial u_{2}}{\partial x_{2}}\right)+\beta_{T} \frac{\partial \theta^{0}}{\partial x_{1}}+f_{1}^{0}=\rho \ddot{u}_{1}^{0}, \\
& \mu \Delta u_{2}+(\lambda+\mu) \frac{\partial}{\partial x_{2}}\left(\frac{\partial u_{1}}{\partial x_{1}}+\frac{\partial u_{2}}{\partial x_{2}}\right)+\beta_{T} \frac{\partial \theta^{0}}{\partial x_{2}}+f_{2}^{0}=\rho \ddot{u}_{2}^{0} \\
& \mu \Delta u_{3}+\mu\left(\frac{\partial \gamma_{1}}{\partial x_{1}}+\frac{\partial \gamma_{2}}{\partial x_{2}}\right)+f_{3}=\rho \ddot{u}_{3}, \\
& \mu \Delta \gamma_{1}+(\lambda+\mu) \frac{\partial}{\partial x_{1}}\left(\frac{\partial \gamma_{1}}{\partial x_{1}}+\frac{\partial \gamma_{2}}{\partial x_{2}}\right)-\frac{3 \mu}{h}\left(\frac{\partial u_{3}}{\partial x_{1}}+\gamma_{1}\right) \\
& +\beta_{T}\left(\frac{\partial \theta^{0}}{\partial x_{1}}+\frac{\partial \theta^{1}}{\partial x_{1}}\right)+f_{1}^{1}=\rho \ddot{\gamma}_{1}, \\
& \mu \Delta \gamma_{2}+(\lambda+\mu) \frac{\partial}{\partial x_{2}}\left(\frac{\partial \gamma_{1}}{\partial x_{1}}+\frac{\partial \gamma_{2}}{\partial x_{2}}\right)-\frac{3 \mu}{h}\left(\frac{\partial u_{3}}{\partial x_{2}}+\gamma_{2}\right) \\
& +\beta_{T}\left(\frac{\partial \theta^{0}}{\partial x_{2}}+\frac{\partial \theta^{1}}{\partial x_{2}}\right)+f_{2}^{1}=\rho \ddot{\gamma}_{2} .
\end{aligned}
$$

For Kirchhoff theory the differential equations in displacements have the form

$$
\begin{gathered}
\mu \Delta u_{1}+(\lambda+\mu) \frac{\partial}{\partial x_{1}}\left(\frac{\partial u_{1}}{\partial x_{1}}+\frac{\partial u_{2}}{\partial x_{2}}\right)+\beta_{T} \frac{\partial \theta^{0}}{\partial x_{1}}+f_{1}=\rho \ddot{u}_{1}^{0}, \\
\mu \Delta u_{2}+(\lambda+\mu) \frac{\partial}{\partial x_{2}}\left(\frac{\partial u_{1}}{\partial x_{1}}+\frac{\partial u_{2}}{\partial x_{2}}\right)+\beta_{T} \frac{\partial \theta^{0}}{\partial x_{2}}+f_{2}=\rho \ddot{u}_{2}^{0}, \\
\mu \Delta \Delta u_{3}+\beta_{T} \Delta \theta^{1}+f_{3}=\rho \ddot{u}_{3} .
\end{gathered}
$$

System of differential equations for Vekua's theory (60) is coupled, and all equations have to be solved together. In Timoshenko's and Kirchhoff's theories the first two equations are independent and can be solved separately. Also in Timoshenko's theory equations for the plate deflection $u_{3}$ and angles of rotation $\gamma_{\alpha}$ are coupled (61) whereas in Kirchhoff's theory we have only one equation for the plate deflection $u_{3}$ of the from (62).

System of differential equations of the heat conductivity is the same for all plate theories considered here and has the form (42), where differential operators have simpler form in Cartesian system of coordinates.

7.2. Equations in the Form of Displacements for Cylindrical Shells. All equations that specify thermodynamical state of the cylindrical shell can be obtained from general equations presented here. In this case one has to introduce cylindrical coordinates in the following way: $x_{1}=r \xi, x_{2}=r \vartheta$, and substitute $A_{1}=A_{2}=r, k_{1}=0, k_{2}=r^{-1}$. After that all corresponding equations for the cylindrical shell theory can be easily obtained. Differential equations in displacements for cylindrical shells have the same structure as in general case but differential operators are much simpler. We present them here.

For Vekua's theory differential operators have the form

$$
\begin{gathered}
L_{11}^{00}=\mu \Delta+(\lambda+\mu) \frac{\partial^{2}}{\partial \xi^{2}}, \\
L_{12}^{00}=(\lambda+\mu) \frac{\partial^{2}}{\partial \xi \partial \vartheta}, \\
L_{13}^{00}=\lambda \frac{\partial}{\partial \xi}, \quad L_{11}^{01}=0, \quad L_{12}^{01}=0, \\
L_{13}^{01}=\lambda \frac{r}{h} \frac{\partial}{\partial \xi}, \quad L_{21}^{00}=(\lambda+\mu) \frac{\partial^{2}}{\partial \xi \partial \vartheta}, \\
L_{22}^{00}=\mu \Delta+(\lambda+\mu) \frac{\partial^{2}}{\partial \vartheta^{2}}-\mu, \\
L_{23}^{00}=(\lambda+2 \mu) \frac{\partial}{\partial \vartheta}+\mu \frac{\partial}{\partial \vartheta}, \\
L_{31}^{01}=-\mu \frac{r}{h} \frac{\partial}{\partial \xi}, \quad L_{32}^{01}=-\mu \frac{r}{h} \frac{\partial}{\partial \vartheta}, \\
L_{22}^{01}=\frac{\mu r}{h}, \\
L_{23}^{01}=\lambda \frac{r}{h} \frac{\partial}{\partial \vartheta}, \quad L_{31}^{00}=-\lambda \frac{\partial}{\partial \xi}, \\
L_{32}^{00}=-\mu \frac{\partial}{\partial \vartheta}-(\lambda+2 \mu) \frac{\partial}{\partial \vartheta}, \\
L_{33}
\end{gathered}
$$




$$
\begin{gathered}
L_{33}^{01}=-\lambda \frac{r}{h}, \quad L_{11}^{10}=0 \\
L_{12}^{10}=0, \quad L_{13}^{10}=-\mu \frac{3 r}{h} \frac{\partial}{\partial \xi} \\
L_{11}^{11}=L_{11}^{00}-\mu \frac{3 r^{2}}{h^{2}}, \\
L_{12}^{11}=L_{12}^{00}, \quad L_{13}^{11}=L_{13}^{00}, \\
L_{21}^{10}=0, \quad L_{22}^{10}=\mu \frac{3 r}{h}, \\
L_{23}^{10}=-\mu \frac{3 r}{h} \frac{\partial}{\partial \vartheta}, \quad L_{21}^{11}=L_{12}^{00}, \\
L_{22}^{11}=L_{22}^{00}-\mu \frac{3 r^{2}}{h^{2}}, \quad L_{23}^{11}=L_{23}^{00}, \\
L_{31}^{10}=-\lambda \frac{3 r}{h} \frac{\partial}{\partial \xi}, \quad L_{32}^{10}=-\lambda \frac{r}{h} \frac{\partial}{\partial \vartheta}, \\
L_{33}^{11}=L_{33}^{00}-(\lambda+2 \mu) \frac{3 r^{2}}{h^{2}} . \\
L_{31}^{10}, \quad \frac{3 r}{h}, \quad L_{31}^{11}=L_{32}^{00},
\end{gathered}
$$
form

For Timoshenko's theory differential operators have the

$$
\begin{gathered}
L_{11}^{00}=D_{n}\left(\frac{\partial^{2}}{\partial \xi^{2}}+\frac{1-v}{2} \frac{\partial^{2}}{\partial \vartheta^{2}}\right), \\
L_{12}^{00}=L_{21}^{00}=D_{n} \frac{1+v}{2} \frac{\partial^{2}}{\partial \xi \partial \vartheta}, \\
L_{13}^{00}=L_{31}^{00}=D_{n} v \frac{\partial}{\partial \xi}, \\
L_{11}^{01}=L_{11}^{10}=L_{12}^{01}=L_{11}^{10}=0 \\
L_{22}^{00}=D_{n}\left(\frac{1-v}{2} \frac{\partial^{2}}{\partial \xi^{2}}+\frac{\partial^{2}}{\partial \vartheta^{2}}-\frac{1-v}{2}\right), \\
L_{21}^{01}=L_{21}^{10}=0, \\
L_{23}^{00}=-L_{32}^{00}=D_{n}\left(\frac{\partial}{\partial \xi}+\frac{1-v}{2} \frac{\partial}{\partial \vartheta}\right), \\
L_{33}^{00}=D_{n} r^{2}\left(\frac{1-v}{2} \Delta-\frac{1}{r^{2}}\right), \\
L_{22}^{01}=L_{22}^{10}=\frac{E h r}{1+v} \\
L_{31}^{01}=-L_{13}^{10}=\frac{E h r}{1+v} \frac{\partial}{\partial \xi} \\
10
\end{gathered}
$$

$$
\begin{gathered}
L_{12}^{11}=L_{21}^{11}=D_{m} \frac{1+v}{2} \frac{\partial^{2}}{\partial \xi \partial \vartheta}, \\
L_{11}^{11}=D_{m}\left(\frac{\partial^{2}}{\partial \xi^{2}}+\frac{1-v}{2} \frac{\partial^{2}}{\partial \vartheta^{2}}\right), \\
L_{22}^{11}=D_{m}\left(\frac{1-v}{2} \frac{\partial^{2}}{\partial \xi^{2}}+\frac{\partial^{2}}{\partial \vartheta^{2}}\right) .
\end{gathered}
$$

Here $D_{n}=2 E h /\left(1-v^{2}\right)$ is a stiffness that corresponds to inplane deformation, and $D_{m}=2 E h^{3} /\left(3\left(1-v^{2}\right)\right)$ is a stiffness that corresponds to bending deformation.

For Kirchhoff-Love's theory differential operators have the form

$$
\begin{gathered}
L_{11}=D_{n}\left(\frac{\partial^{2}}{\partial \xi^{2}}+\frac{1-v}{2} \frac{\partial^{2}}{\partial \vartheta^{2}}\right) \\
L_{12}=L_{21}=D_{n} \frac{1+v}{2} \frac{\partial^{2}}{\partial \xi \partial \vartheta} \\
L_{13}=-L_{31}=D_{n} v \frac{\partial}{\partial \xi} \\
L_{22}=D_{n}\left(\frac{1-v}{2} \frac{\partial^{2}}{\partial \xi^{2}}+\frac{\partial^{2}}{\partial \vartheta^{2}}-\frac{1-v}{2}\right) \\
+D_{m} \frac{1}{r^{2}}\left(2(1-v) \frac{\partial^{2}}{\partial \xi^{2}}+\frac{\partial^{2}}{\partial \vartheta^{2}}\right), \\
L_{23}=-L_{32}=D_{n} \frac{\partial}{\partial \vartheta}-D_{m} \frac{1}{r^{2}}\left((2-v) \frac{\partial^{3}}{\partial \xi^{2} \partial \vartheta}+\frac{\partial^{3}}{\partial \xi^{3}}\right), \\
L_{33}=-D_{n}-D_{m} \Delta \Delta .
\end{gathered}
$$

System of differential equations of the heat conductivity is the same for all cylindrical shell theories considered here and has the form (42), where differential operators have simpler form in cylindrical system of coordinates.

\section{Conclusions}

In this paper a high order theory for homogeneous thermoelastic shells has been developed. The proposed approach is based on expansion of the coupled equations of thermoelasticity and heat conductivity into Fourier series in terms of Legendre polynomials. Starting from the equations of coupled thermoelasticity and heat conductivity, stress and strain tensors, vectors of displacements, traction and body forces, temperature, and vector of thermal flow have been expanded into Fourier series in terms of Legendre polynomials in a thickness coordinate. Thereby all equations of thermoelasticity including generalized Hooke's law and equations of heat conductivity have been transformed to corresponding equations for Fourier coefficients of Legendre's polynomial expansion. The system of differential equations in terms of displacements including initial and boundary 
conditions for Fourier coefficients has been obtained. Special attention has been paid to the case of the first approximation shells theory. All equations of thermoelasticity and heat conductivity for Vekua's, Timoshenko's, and Kirchhoff-Love's shell theories have been obtained. Differential operator for shells of arbitrary geometry, cylindrical shells, and plates has been obtained and presented in the explicit form. Comparison of Vekua's, Timoshenko's, and Kirchhoff-Love's shell theories has been done, and corresponding equations have been analyzed. Obtained equations can be used for thermomechanical analysis of the shells of any specific geometry and also for numerical calculations.

\section{Appendix}

For high order shell theory

$$
\begin{aligned}
& L_{11}^{k(k-i)} u_{1}^{k-i} \\
& =\left\{\begin{array}{l}
\mu A_{1} A_{2} k_{1} \frac{2 k+1}{h} u_{1}^{k-i} \\
\quad \text { for } i<k, \text { odd } i \\
-\mu A_{1} A_{2} \frac{2 k+1}{h^{2}} \\
\times((2(k-i)-1)+(2(k-i)-5)+\cdots) u_{1}^{k-i} \\
\quad \text { for } i<k, \text { even } i,
\end{array}\right. \\
& L_{11}^{k k} u_{1}^{k} \\
& =\mu A_{1} A_{2} \Delta u_{1}^{k}+(\lambda+\mu) \frac{\partial}{\partial x_{1}}\left(\frac{A_{2}}{A_{1}} \frac{\partial u_{1}^{k}}{\partial x_{1}}\right) \\
& -\mu\left(\frac{\partial}{\partial x_{2}}\left(\frac{u_{1}^{k}}{A_{2}} \frac{\partial A_{1}}{\partial x_{2}}\right)+\frac{u_{1}^{k}}{A_{1} A_{2}}\left(\frac{\partial A_{2}}{\partial x_{2}}\right)^{2}\right. \\
& \left.+A_{1} A_{2} k_{1}^{2} u_{1}^{k}\right) \\
& -(\lambda+2 \mu) \frac{u_{1}^{k}}{A_{1} A_{2}}\left(\frac{\partial A_{2}}{\partial x_{1}}\right)^{2} \\
& -\lambda \frac{1}{A_{1}} \frac{\partial A_{2}}{\partial x_{1}} \frac{\partial u_{1}^{k}}{\partial x_{1}}-A_{1} A_{2} \mu \frac{2 k+1}{h^{2}} \\
& \times((2 k-1)+(2 k-5)+\cdots) u_{1}^{k} \text {, } \\
& L_{11}^{k(k+i)} u_{1}^{k+i} \\
& =\left\{\begin{array}{l}
\mu A_{1} A_{2} k_{1} \frac{2 k+1}{h} u_{1}^{k+i} \\
\quad \text { for } i>k, \text { odd } i \\
-\mu A_{1} A_{2} \frac{2 k+1}{h^{2}} \\
\quad \times((2 k-1)+(2 k-5)+\cdots) u_{1}^{k+i} \\
\quad \text { for } i>k, \text { even } i,
\end{array}\right.
\end{aligned}
$$

$$
\begin{aligned}
& L_{12}^{k k} u_{2}^{k} \\
& =(\lambda+2 \mu)\left(\frac{\partial}{\partial x_{1}}\left(\frac{u_{2}^{k}}{A_{1}} \frac{\partial A_{1}}{\partial x_{2}}\right)-\frac{1}{A_{2}} \frac{\partial A_{2}}{\partial x_{1}} \frac{\partial u_{2}^{k}}{\partial x_{2}}\right) \\
& +(\lambda+\mu) \frac{\partial^{2} u_{2}^{k}}{\partial x_{1} \partial x_{2}}-\lambda \frac{u_{2}^{k}}{A_{1} A_{2}} \frac{\partial A_{1}}{\partial x_{2}} \frac{\partial A_{2}}{\partial x_{1}} \\
& +\mu\left(\frac{\partial A_{1}}{\partial x_{2}} \frac{\partial A_{2}}{\partial x_{1}} \frac{u_{2}^{k}}{A_{1} A_{2}}+\frac{1}{A_{1}} \frac{\partial A_{1}}{\partial x_{2}} \frac{\partial u_{2}^{k}}{\partial x_{1}}\right. \\
& \left.-\frac{\partial}{\partial x_{2}}\left(\frac{u_{2}^{k}}{A_{2}} \frac{\partial A_{2}}{\partial x_{1}}\right)\right),
\end{aligned}
$$$$
L_{13}^{k(k-i)} u_{3}^{k-i}= \begin{cases}-\mu A_{2} \frac{2 k+1}{h} \frac{\partial u_{3}^{k-i}}{\partial x_{1}} & \text { for } i<k, \text { odd } i \\ 0 & \text { for } i<k, \text { even } i\end{cases}
$$$$
L_{13}^{k k} u_{3}^{k}
$$$$
=(\lambda+2 \mu)\left(\frac{\partial\left(A_{1} k_{1} u_{3}^{k}\right)}{\partial x_{1}}-k_{2} \frac{\partial A_{2}}{\partial x_{1}} u_{3}^{k}\right)
$$$$
+\lambda\left(\frac{\partial\left(A_{2} k_{2} u_{3}^{k}\right)}{\partial x_{1}}-k_{1} \frac{\partial A_{2}}{\partial x_{1}} u_{3}^{k}\right)
$$$$
+\mu A_{2} k_{1} \frac{\partial u_{3}^{k}}{\partial x_{1}}
$$$$
L_{13}^{k(k+i)} u_{3}^{k+i}
$$$$
= \begin{cases}\lambda A_{2} \frac{2 k+1}{h} \frac{\partial u_{3}^{k+i}}{\partial x_{1}} & \text { for } i>k, \text { odd } i \\ 0 & \text { for } i>k, \text { even } i,\end{cases}
$$$$
L_{21}^{k k} u_{1}^{k}
$$$$
=(\lambda+2 \mu)\left(\frac{\partial}{\partial x_{2}}\left(\frac{u_{1}^{k}}{A_{2}} \frac{\partial A_{2}}{\partial x_{1}}\right)-\frac{1}{A_{1}} \frac{\partial A_{1}}{\partial x_{2}} \frac{\partial u_{1}^{k}}{\partial x_{1}}\right)
$$$$
+(\lambda+\mu) \frac{\partial^{2} u_{1}^{k}}{\partial x_{1} \partial x_{2}}-\lambda \frac{1}{A_{1} A_{2}} \frac{\partial A_{2}}{\partial x_{1}} \frac{\partial A_{2}}{\partial x_{1}} u_{1}^{k}
$$$$
+\mu\left(\frac{1}{A_{2}} \frac{\partial A_{2}}{\partial x_{1}} \frac{\partial u_{1}^{k}}{\partial x_{2}}-\frac{u_{1}^{k}}{A_{1} A_{2}} \frac{\partial A_{2}}{\partial x_{1}} \frac{\partial A_{1}}{\partial x_{2}}\right.
$$$$
\left.-\frac{\partial}{\partial x_{1}}\left(\frac{u_{1}^{k}}{A_{1}} \frac{\partial A_{1}}{\partial x_{2}}\right)\right)
$$

$$
\begin{aligned}
& L_{22}^{k(k-i)} u_{1}^{k-i} \\
& =\left\{\begin{array}{c}
\mu A_{1} A_{2} k_{2} \frac{2 k+1}{h} u_{2}^{k-i} \\
\quad \text { for } i<k, \text { odd } i \\
-\mu A_{1} A_{2} \frac{2 k+1}{h^{2}} \\
\quad \times((2(k-i)-1)+(2(k-i)-5)+\cdots) u_{2}^{k-i} \\
\quad \text { for } i<k, \text { even } i,
\end{array}\right.
\end{aligned}
$$




$$
\begin{aligned}
& L_{22}^{k k} u_{2}^{k} \\
& =\mu A_{1} A_{2} \Delta u_{2}^{k}+(\lambda+\mu) \frac{\partial}{\partial x_{2}}\left(\frac{A_{1}}{A_{2}} \frac{\partial u_{2}^{k}}{\partial x_{2}}\right) \\
& -\mu\left(\frac{\partial}{\partial x_{1}}\left(\frac{u_{2}^{k}}{A_{1}} \frac{\partial A_{2}}{\partial x_{1}}\right)+\frac{u_{2}^{k}}{A_{1} A_{2}}\left(\frac{\partial A_{2}}{\partial x_{2}}\right)^{2}\right. \\
& \left.+A_{1} A_{2} k_{2}^{2} u_{2}^{k}\right) \\
& -(\lambda+2 \mu) \frac{u_{2}^{k}}{A_{1} A_{2}}\left(\frac{\partial A_{1}}{\partial x_{2}}\right)^{2} \\
& -\lambda \frac{1}{A_{2}} \frac{\partial A_{1}}{\partial x_{2}} \frac{\partial u_{2}^{k}}{\partial x_{2}}-A_{1} A_{2} \mu \frac{2 k+1}{h^{2}} \\
& \times((2 k-1)+(2 k-5)+\cdots) u_{2}^{k}, \\
& L_{22}^{k(k+i)} u_{2}^{k+i} \\
& =\left\{\begin{array}{l}
\mu A_{1} A_{2} k_{2} \frac{2 k+1}{h} u_{2}^{k+i} \\
\quad \text { for } i<k \text {, odd } i \\
-\mu A_{1} A_{2} \frac{2 k+1}{h^{2}} \\
\times((2 k-1)+(2 k-5)+\cdots) u_{2}^{k+i} \\
\quad \text { for } i<k, \text { even } i,
\end{array}\right. \\
& L_{23}^{k(k-i)} u_{3}^{k-i}= \begin{cases}-\mu A_{1} \frac{2 k+1}{h} \frac{\partial u_{3}^{k-i}}{\partial x_{2}} & \text { for } i<k, \text { odd } i \\
0 & \text { for } i<k, \text { even } i\end{cases} \\
& L_{23}^{k k} u_{3}^{k} \\
& =(\lambda+2 \mu)\left(\frac{\partial\left(A_{2} k_{2} u_{3}^{k}\right)}{\partial x_{2}}-k_{1} \frac{\partial A_{1}}{\partial x_{2}} u_{3}^{k}\right) \\
& +\lambda\left(\frac{\partial\left(A_{1} k_{1} u_{3}^{k}\right)}{\partial x_{2}}-k_{2} \frac{\partial A_{1}}{\partial x_{2}} u_{3}^{k}\right)+\mu A_{1} k_{2} \frac{\partial u_{3}^{k}}{\partial x_{2}}, \\
& L_{23}^{k(k+i)} u_{3}^{k+i} \\
& = \begin{cases}\lambda A_{2} \frac{2 k+1}{h} \frac{\partial u_{3}^{k+i}}{\partial x_{2}} & \text { for } i<k, \text { odd } i \\
0 & \text { for } i<k, \text { even } i,\end{cases} \\
& L_{31}^{k(k-i)} u_{1}^{k-i} \\
& = \begin{cases}-\lambda \frac{2 k+1}{h} \frac{\partial\left(A_{2} u_{1}^{k-i}\right)}{\partial x_{1}} & \text { for } i<k, \text { odd } i \\
0 & \text { for } i<k, \text { even } i,\end{cases} \\
& L_{31}^{k k} u_{1}^{k} \\
& =-\mu \frac{\partial\left(A_{1} k_{1} u_{1}^{k}\right)}{\partial x_{1}}-(\lambda+2 \mu)
\end{aligned}
$$

$$
\begin{aligned}
& \times\left(u_{1}^{k} k_{2} \frac{\partial A_{2}}{\partial x_{1}}+k_{1} A_{2} \frac{\partial u_{1}^{k}}{\partial x_{1}}\right) \\
& -\lambda\left(k_{1} u_{1}^{k} \frac{\partial A_{2}}{\partial x_{1}}+k_{2} A_{2} \frac{\partial u_{1}^{k}}{\partial x_{1}}\right),
\end{aligned}
$$$$
L_{31}^{k(k+i)} u_{1}^{k+i}= \begin{cases}\mu \frac{2 k+1}{h} \frac{\partial\left(A_{2} u_{1}^{k+i}\right)}{\partial x_{1}} & \text { for } i<k, \text { odd } i \\ 0 & \text { for } i<k, \text { even } i,\end{cases}
$$$$
L_{32}^{k(k-i)} u_{2}^{k-i}= \begin{cases}-\lambda \frac{2 k+1}{h} \frac{\partial\left(A_{1} u_{2}^{k-i}\right)}{\partial x_{2}} & \text { for } i<k, \text { odd } i \\ 0 & \text { for } i<k, \text { even } i,\end{cases}
$$$$
L_{31}^{k k} u_{1}^{k}
$$$$
=-\mu \frac{\partial\left(A_{2} k_{2} u_{2}^{k}\right)}{\partial x_{2}}-(\lambda+2 \mu)
$$$$
\times\left(u_{1}^{k} k_{1} \frac{\partial A_{1}}{\partial x_{2}}+k_{2} A_{1} \frac{\partial u_{2}^{k}}{\partial x_{2}}\right)
$$$$
-\lambda\left(k_{2} u_{2}^{k} \frac{\partial A_{1}}{\partial x_{2}}+k_{1} A_{1} \frac{\partial u_{2}^{k}}{\partial x_{2}}\right),
$$$$
L_{32}^{k(k+i)} u_{2}^{k+i}= \begin{cases}\mu \frac{2 k+1}{h} \frac{\partial\left(A_{1} u_{2}^{k+i}\right)}{\partial x_{2}} & \text { for } i<k, \text { odd } i \\ 0 & \text { for } i<k, \text { even } i,\end{cases}
$$$$
L_{33}^{k(k-i)} u_{3}^{k-i}
$$$$
=\left\{\begin{array}{l}
-\lambda A_{1} A_{2}\left(k_{1}+k_{2}\right) \frac{2 k+1}{h} u_{3}^{k-i} \\
\quad \text { for } i<k, \text { odd } i \\
(\lambda+2 \mu) A_{1} A_{2} \frac{2 k+1}{h^{2}} \\
\quad \times((2(k-i)-1)+(2(k-i)-5)+\cdots) u_{3}^{k-i} \\
\quad \text { for } i<k, \text { even } i,
\end{array}\right.
$$$$
L_{33}^{k k} u_{3}^{k}
$$$$
=\mu A_{1} A_{2} \Delta u_{3}^{k}-(\lambda+2 \mu)\left(k_{1}^{2}+k_{2}^{2}\right) u_{3}^{k}
$$$$
-(\lambda+2 \mu) A_{1} A_{2} \frac{2 k+1}{h^{2}}
$$$$
\times((2 k-1)+(2 k-5)+\cdots) u_{3}^{k}
$$$$
-2 \lambda A_{1} A_{2} k_{1} k_{2} u_{3}^{k} \text {, }
$$

$$
L_{33}^{k(k+i)} u_{3}^{k+i}
$$$$
=\left\{\begin{array}{l}
-\lambda A_{1} A_{2}\left(k_{1}+k_{2}\right) \frac{2 k+1}{h} u_{3}^{k+i} \\
\quad \text { for } i<k, \text { odd } i \\
(\lambda+2 \mu) A_{1} A_{2} \frac{2 k+1}{h^{2}} \\
\quad \times((2 k-1)+(2 k-5)+\cdots) u_{3}^{k+i} \\
\quad \text { for } i<k, \text { even } i,
\end{array}\right.
$$ 


$$
\begin{gathered}
L_{12}^{k(k-i)} u_{2}^{k-i}=0, \quad L_{12}^{k(k+i)} u_{2}^{k+i}=0, \\
L_{21}^{k(k-i)} u_{1}^{k-i}=0, \quad L_{21}^{k(k+i)} u_{1}^{k+i}=0, \\
L_{1}^{k} \theta^{k}=-\beta_{\tau} A_{1} \frac{\partial\left(\theta^{k}-\theta_{0}^{k}\right)}{\partial x_{1}}, \\
L_{2}^{k} \theta^{k}=-\beta_{\tau} A_{1} \frac{\partial\left(\theta^{k}-\theta_{0}^{k}\right)}{\partial x_{2}}, \\
L_{3}^{k} \theta^{k}=A_{1} A_{2}\left(k_{1}+k_{2}\right) \beta_{\tau}\left(\theta^{k}-\theta_{0}^{k}\right)+2 \beta_{\tau} Q_{3}^{k},
\end{gathered}
$$

where

$$
\begin{aligned}
& \Delta=\frac{1}{A_{1} A_{2}}\left(\frac{\partial}{\partial x_{1}}\left(\frac{A_{2}}{A_{1}} \frac{\partial}{\partial x_{1}}\right)+\frac{\partial}{\partial x_{2}}\left(\frac{A_{1}}{A_{2}} \frac{\partial}{\partial x_{2}}\right)\right), \\
& \underline{\sigma_{13}^{k}} \\
& =A_{1} A_{2} \frac{2 k+1}{h} \\
& \times\left(\mu\left(\frac{1}{A_{1}} \frac{\partial u_{3}^{k-1}}{\partial x_{1}}-k_{1} u_{1}^{k-1}+\underline{u_{1}^{k-1}}\right)\right. \\
& \left.+\mu\left(\frac{1}{A_{1}} \frac{\partial u_{3}^{k-3}}{\partial x_{1}}-k_{1} u_{1}^{k-3}+\underline{u_{1}^{k-3}}\right)+\cdots\right), \\
& \underline{\sigma_{23}^{k}} \\
& =A_{1} A_{2} \frac{2 k+1}{h} \\
& \times\left(\mu\left(\frac{1}{A_{2}} \frac{\partial u_{3}^{k-1}}{\partial x_{2}}-k_{2} u_{2}^{k-1}+\underline{u_{2}^{k-1}}\right)\right. \\
& \left.+\mu\left(\frac{1}{A_{2}} \frac{\partial u_{3}^{k-3}}{\partial x_{2}}-k_{2} u_{2}^{k-3}+\underline{u_{2}^{k-3}}\right)+\cdots\right), \\
& =A_{1} A_{2} \frac{2 k+1}{h}\left((\lambda+2 \mu) \underline{u_{3}^{k-1}}\right. \\
& +\lambda\left(\left(\frac{1}{A_{1}} \frac{\partial u_{1}^{k-1}}{\partial x_{1}}+\frac{1}{A_{1} A_{2}} \frac{\partial A_{1}}{\partial x_{2}} u_{2}^{k-1}+k_{1} u_{3}^{k-1}\right)\right. \\
& \left.+\left(\frac{1}{A_{2}} \frac{\partial u_{2}^{k-1}}{\partial x_{2}}+\frac{1}{A_{1} A_{2}} \frac{\partial A_{2}}{\partial x_{1}} u_{1}^{k-1}\right)\right) \\
& \left.+\sigma_{33}^{k-3}+\cdots\right) \\
& \underline{u_{i}^{k}}=\frac{2 k+1}{h}\left(u_{i}^{k+1}+u_{i}^{k+3}+\cdots\right) .
\end{aligned}
$$

For Vekua's shell theory

$$
\begin{aligned}
& L_{11}^{00} u_{1}^{0} \\
& =\mu A_{1} A_{2} \Delta\left(u_{1}^{0}\right)+(\lambda+\mu) \frac{\partial}{\partial x_{1}}\left(\frac{A_{2}}{A_{1}} \frac{\partial u_{1}^{0}}{\partial x_{1}}\right) \\
& -\mu\left[\frac{\partial}{\partial x_{2}}\left(\frac{1}{A_{2}} \frac{\partial A_{1}}{\partial x_{2}}\right)+\frac{1}{A_{1} A_{2}}\left(\frac{\partial A_{1}}{\partial x_{2}}\right)^{2}+A_{1} A_{2} k_{1}^{2}\right] u_{1}^{0} \\
& -(\lambda+2 \mu) \frac{1}{A_{1} A_{2}}\left(\frac{\partial A_{2}}{\partial x_{1}}\right)^{2} u_{1}^{0}-\lambda \frac{1}{A_{1}}\left(\frac{\partial A_{2}}{\partial x_{1}}\right) \frac{\partial u_{1}^{0}}{\partial x_{1}}, \\
& L_{12}^{00} u_{2}^{0}
\end{aligned}
$$

$$
\begin{gathered}
=(\lambda+2 \mu)\left[\frac{\partial}{\partial x_{1}}\left(\frac{1}{A_{1}} \frac{\partial A_{1}}{\partial x_{2}} u_{2}^{0}\right)-\frac{1}{A_{2}} \frac{\partial A_{1}}{\partial x_{2}} \frac{\partial u_{2}^{0}}{\partial x_{2}}\right] \\
+(\lambda+\mu) \frac{\partial^{2} u_{2}^{0}}{\partial x_{1} \partial x_{2}}-\lambda \frac{1}{A_{1} A_{2}} \frac{\partial A_{1}}{\partial x_{2}} \frac{\partial A_{2}}{\partial x_{2}} u_{2}^{0} \\
-\mu\left[\frac{\partial}{\partial x_{2}}\left(\frac{1}{A_{2}} \frac{\partial A_{2}}{\partial x_{1}} u_{2}^{0}\right)\right. \\
\left.-\frac{1}{A_{1} A_{2}} \frac{\partial A_{1}}{\partial x_{2}} \frac{\partial A_{2}}{\partial x_{2}} u_{2}^{0}-\frac{1}{A_{1}} \frac{\partial A_{1}}{\partial x_{2}} \frac{\partial u_{2}^{0}}{\partial x_{1}}\right]
\end{gathered}
$$

$L_{13}^{00} u_{3}^{0}$

$$
\begin{gathered}
=(\lambda+2 \mu)\left[\frac{\partial}{\partial x_{1}}\left(A_{1} k_{1} u_{3}^{0}\right)-k_{2} \frac{\partial A_{2}}{\partial x_{1}} u_{3}^{0}\right] \\
+\lambda\left[\frac{\partial}{\partial x_{1}}\left(A_{2} k_{2} u_{3}^{0}\right)-k_{1} \frac{\partial A_{2}}{\partial x_{1}} u_{3}^{0}\right]+\mu A_{2} k_{1} \frac{\partial u_{3}^{0}}{\partial x_{1}}, \\
L_{11}^{01} u_{1}^{1}=\mu A_{1} A_{2} k_{1} \frac{u_{1}^{1}}{h}, \quad L_{12}^{01} u_{2}^{1}=0, \\
L_{13}^{01} u_{3}^{1}=\lambda A_{2} \frac{1}{h} \frac{\partial u_{3}^{1}}{\partial x_{1}},
\end{gathered}
$$

$L_{21}^{00} u_{1}^{0}$

$$
\begin{gathered}
=(\lambda+2 \mu)\left[\frac{\partial}{\partial x_{2}}\left(\frac{1}{A_{2}} \frac{\partial A_{2}}{\partial x_{1}} u_{1}^{0}\right)-\frac{1}{A_{1}} \frac{\partial A_{1}}{\partial x_{2}} \frac{\partial u_{1}^{0}}{\partial x_{1}}\right] \\
+(\lambda+\mu) \frac{\partial^{2} u_{1}^{0}}{\partial x_{1} \partial x_{2}}-\lambda \frac{1}{A_{1} A_{2}} \frac{\partial A_{1}}{\partial x_{2}} \frac{\partial A_{2}}{\partial x_{2}} u_{1}^{0} \\
-\mu\left[\frac{\partial}{\partial x_{1}}\left(\frac{1}{A_{1}} \frac{\partial A_{1}}{\partial x_{2}} u_{1}^{0}\right)\right. \\
\left.\quad-\frac{1}{A_{1} A_{2}} \frac{\partial A_{2}}{\partial x_{1}} \frac{\partial A_{1}}{\partial x_{2}} u_{1}^{0}-\frac{1}{A_{2}} \frac{\partial A_{2}}{\partial x_{1}} \frac{\partial u_{1}^{0}}{\partial x_{2}}\right] \\
L_{22}^{00} u_{1}^{0} \quad \\
=\mu A_{1} A_{2} \Delta\left(u_{2}^{0}\right)+(\lambda+\mu) \frac{\partial}{\partial x_{2}}\left(\frac{A_{1}}{A_{2}} \frac{\partial u_{2}^{0}}{\partial x_{2}}\right)
\end{gathered}
$$




$$
\begin{aligned}
& -\mu\left[\frac{\partial}{\partial x_{1}}\left(\frac{1}{A_{1}} \frac{\partial A_{2}}{\partial x_{1}}\right)+\frac{1}{A_{1} A_{2}}\left(\frac{\partial A_{2}}{\partial x_{1}}\right)^{2}+A_{1} A_{2} k_{2}^{2}\right] u_{2}^{0} \\
& -(\lambda+2 \mu) \frac{1}{A_{1} A_{2}}\left(\frac{\partial A_{1}}{\partial x_{2}}\right)^{2} u_{2}^{0}-\lambda \frac{1}{A_{2}}\left(\frac{\partial A_{1}}{\partial x_{2}}\right) \frac{\partial u_{2}^{0}}{\partial x_{2}}, \\
& L_{23}^{00} u_{3}^{0} \\
& =(\lambda+2 \mu)\left[\frac{\partial}{\partial x_{2}}\left(A_{2} k_{2} u_{3}^{0}\right)-k_{1} \frac{\partial A_{1}}{\partial x_{2}} u_{3}^{0}\right] \\
& +\lambda\left[\frac{\partial}{\partial x_{2}}\left(A_{1} k_{1} u_{3}^{0}\right)-k_{2} \frac{\partial A_{1}}{\partial x_{2}} u_{3}^{0}\right]+\mu A_{1} k_{2} \frac{\partial u_{3}^{0}}{\partial x_{2}} \\
& L_{21}^{01} u_{1}^{1}=0, \quad L_{22}^{01} u_{2}^{1}=\mu A_{1} A_{2} k_{2} \frac{u_{2}^{1}}{h}, \\
& L_{23}^{01} u_{3}^{1}=\lambda A_{2} \frac{1}{h} \frac{\partial u_{3}^{1}}{\partial x_{2}}, \\
& L_{31}^{00} u_{1}^{0} \\
& =-\mu \frac{\partial}{\partial x_{1}}\left(A_{1} k_{1} u_{1}^{0}\right)-(\lambda+2 \mu) \\
& \times\left[k_{2} \frac{\partial A_{2}}{\partial x_{1}} u_{1}^{0}+k_{1} A_{2} \frac{\partial u_{1}^{0}}{\partial x_{1}}\right] \\
& -\lambda\left[k_{1} \frac{\partial A_{2}}{\partial x_{1}} u_{1}^{0}+A_{2} k_{2} \frac{\partial u_{1}^{0}}{\partial x_{1}}\right], \\
& L_{32}^{00} u_{2}^{0} \\
& =-\mu \frac{\partial}{\partial x_{2}}\left(A_{2} k_{2} u_{2}^{0}\right)-(\lambda+2 \mu) \\
& \times\left[k_{1} \frac{\partial A_{1}}{\partial x_{2}} u_{2}^{0}+k_{2} A_{1} \frac{\partial u_{2}^{0}}{\partial x_{2}}\right] \\
& -\lambda\left[k_{2} \frac{\partial A_{1}}{\partial x_{2}} u_{2}^{0}+A_{1} k_{1} \frac{\partial u_{2}^{0}}{\partial x_{2}}\right] \text {, } \\
& L_{33}^{00} u_{3}^{0}=-\mu A_{1} A_{2} \Delta u_{3}^{0}-(\lambda+2 \mu) A_{1} A_{2}\left(k_{1}^{2}+k_{2}^{2}\right) u_{3}^{0} \\
& -2 \lambda A_{1} A_{2} k_{1} k_{2} u_{3}^{0} \\
& L_{31}^{01} u_{1}^{1}=-\mu \frac{1}{h} \frac{\partial\left(A_{2} u_{1}^{1}\right)}{\partial x_{1}}, \quad L_{32}^{01} u_{2}^{1}=-\mu \frac{1}{h} \frac{\partial\left(A_{1} u_{2}^{1}\right)}{\partial x_{2}}, \\
& L_{33}^{01} u_{3}^{1}-\lambda A_{1} A_{2}\left(k_{1}+k_{2}\right) \frac{1}{h} u_{3}^{1} \\
& L_{11}^{10} u_{1}^{0}=\mu A_{1} A_{2} k_{1} \frac{3 u_{1}^{0}}{h}, \quad L_{12}^{10} u_{2}^{0}=0, \\
& L_{13}^{10} u_{3}^{0}=-\mu A_{2} \frac{3}{h} \frac{\partial u_{3}^{0}}{\partial x_{1}} \\
& L_{11}^{11} u_{1}^{1}=L_{11}^{00} u_{1}^{1}-\mu A_{1} A_{2} \frac{3 u_{1}^{1}}{h^{2}}
\end{aligned}
$$

For Timoshenko's shell theory

$$
\begin{aligned}
& L_{11}^{00} u_{1} \\
& =D_{n}\left\{\frac{\partial}{\partial x_{1}}\left(\frac{A_{2}}{A_{1}} \frac{\partial u_{1}}{\partial x_{1}}\right)-\frac{1}{A_{1} A_{2}}\left(\frac{\partial A_{2}}{\partial x_{1}}\right)^{2} u_{1}\right. \\
& +v\left[\frac{\partial}{\partial x_{1}}\left(\frac{1}{A_{1}} \frac{\partial A_{2}}{\partial x_{1}} u_{1}\right)-\frac{1}{A_{1}} \frac{\partial A_{2}}{\partial x_{1}} \frac{\partial u_{1}}{\partial x_{1}}\right]+\frac{1-v}{2} \\
& \times\left[\frac{A_{1}}{A_{2}} \frac{\partial A_{1}}{\partial x_{2}} \frac{\partial}{\partial x_{2}}\left(\frac{u_{1}}{A_{1}}\right)+\frac{\partial}{\partial x_{2}}\right. \\
& \left.\times\left(\frac{A_{1}^{2}}{A_{2}} \frac{\partial}{\partial x_{2}}\left(\frac{u_{1}}{A_{1}}\right)\right)-A_{1} A_{2} k_{1}^{2} u_{1}\right] \\
& +D_{m}\left[\frac{\partial}{\partial x_{2}}\left(\frac{k_{1} k_{2} A_{1}}{A_{2}} \frac{\partial u_{1}}{\partial x_{2}}\right)-\frac{\partial}{\partial x_{2}}\left(\frac{k_{1}^{2}}{A_{2}} \frac{\partial A_{1}}{\partial x_{2}} u_{1}\right)\right. \\
& \left.\left.-\frac{k_{1} k_{2}}{A_{1} A_{2}}\left(\frac{\partial A_{1}}{\partial x_{2}}\right)^{2} u_{1}+\frac{k_{2}^{2}}{A_{2}} \frac{\partial A_{1}}{\partial x_{2}} \frac{\partial u_{1}}{\partial x_{2}}\right]\right\}, \\
& L_{12}^{00} u_{2} \\
& =D_{n}\left\{\frac{\partial}{\partial x_{1}}\left(\frac{1}{A_{1}} \frac{\partial A_{1}}{\partial x_{2}} u_{2}\right)-\frac{1}{A_{2}} \frac{\partial A_{2}}{\partial x_{1}} \frac{\partial u_{2}}{\partial x_{2}}\right. \\
& +v\left(\frac{\partial^{2} u_{2}}{\partial x_{1} \partial x_{2}}-\frac{1}{A_{1} A_{2}} \frac{\partial A_{1}}{\partial x_{2}} \frac{\partial A_{2}}{\partial x_{1}} u_{2}\right)
\end{aligned}
$$




$$
\begin{aligned}
& +\frac{1-v}{2}\left[\frac{\partial}{\partial x_{2}} A_{2} \frac{\partial}{\partial x_{1}}\left(\frac{u_{2}}{A_{2}}\right)\right. \\
& \left.+\frac{A_{2}}{A_{1}} \frac{\partial A_{1}}{\partial x_{2}} \frac{\partial}{\partial x_{1}}\left(\frac{u_{2}}{A_{2}}\right)\right] \\
& +D_{m}\left[\frac{\partial}{\partial x_{2}}\left(k_{1}^{2} \frac{\partial u_{2}}{\partial x_{1}}\right)-\frac{\partial}{\partial x_{2}}\left(\frac{k_{1} k_{2}}{A_{2}} \frac{\partial A_{2}}{\partial x_{1}} u_{2}\right)\right. \\
& \left.\left.+\frac{k_{1} k_{2}}{A_{1}} \frac{\partial A_{1}}{\partial x_{2}} \frac{\partial u_{2}}{\partial x_{1}}-\frac{k_{1}^{2}}{A_{1} A_{2}} \frac{\partial A_{1}}{\partial x_{2}} \frac{\partial A_{2}}{\partial x_{1}} u_{2}\right]\right\}, \\
& L_{13}^{00} u_{3} \\
& =D_{n}\left[\frac{\partial}{\partial x_{1}}\left(A_{2}\left(k_{1}+v k_{2}\right) u_{3}\right)\right. \\
& \left.-\left(k_{2}+v k_{1}\right) \frac{\partial A_{2}}{\partial x_{1}} u_{3}+\frac{1-v}{2} A_{2} k_{1} \frac{\partial u_{3}}{\partial x_{1}}\right], \\
& =D_{m}\left[\frac{\partial}{\partial x_{2}}\left(\frac{A_{1} k_{1}}{A_{2}} \frac{\partial \gamma_{1}}{\partial x_{2}}\right)-\frac{\partial}{\partial x_{2}}\left(\frac{k_{1}}{A_{2}} \frac{\partial A_{1}}{\partial x_{2}} \gamma_{1}\right)\right. \\
& \left.-\frac{k_{2}}{A_{1} A_{2}}\left(\frac{\partial A_{1}}{\partial x_{2}}\right)^{2} \gamma_{1}+\frac{k_{1}}{A_{2}} \frac{\partial A_{1}}{\partial x_{2}} \frac{\partial \gamma_{1}}{\partial x_{2}}\right] \\
& +D_{n} A_{1} A_{2} k_{1} \gamma_{1} \text {, } \\
& L_{12}^{01} \gamma_{2} \\
& =D_{m}\left[\frac{\partial}{\partial x_{2}}\left(k_{1} \frac{\partial \gamma_{2}}{\partial x_{1}}\right)-\frac{\partial}{\partial x_{2}}\left(\frac{k_{1}}{A_{2}} \frac{\partial A_{2}}{\partial x_{1}} \gamma_{2}\right)\right. \\
& \left.-\frac{k_{2}}{A_{1} A_{2}} \frac{\partial A_{1}}{\partial x_{2}} \frac{\partial A_{2}}{\partial x_{1}} \gamma_{2}+\frac{k_{2}}{A_{1}} \frac{\partial A_{1}}{\partial x_{2}} \frac{\partial \gamma_{2}}{\partial x_{1}}\right] \text {, } \\
& =D_{n}\left\{\frac{\partial}{\partial x_{2}}\left(\frac{1}{A_{2}} \frac{\partial A_{2}}{\partial x_{1}} u_{1}\right)-\frac{1}{A_{1}} \frac{\partial A_{1}}{\partial x_{2}} \frac{\partial u_{1}}{\partial x_{1}}\right. \\
& +v\left(\frac{\partial^{2} u_{1}}{\partial x_{1} \partial x_{2}}-\frac{1}{A_{1} A_{2}} \frac{\partial A_{2}}{\partial x_{1}} \frac{\partial A_{1}}{\partial x_{2}} u_{1}\right) \\
& +\frac{1-v}{2}\left[\frac{\partial}{\partial x_{1}} A_{1} \frac{\partial}{\partial x_{2}}\left(\frac{u_{1}}{A_{1}}\right)\right. \\
& \left.+\frac{A_{1}}{A_{2}} \frac{\partial A_{2}}{\partial x_{1}} \frac{\partial}{\partial x_{2}}\left(\frac{u_{1}}{A_{1}}\right)\right] \\
& +D_{m}\left[\frac{\partial}{\partial x_{1}}\left(k_{2}^{2} \frac{\partial u_{1}}{\partial x_{2}}\right)-\frac{\partial}{\partial x_{1}}\left(\frac{k_{1} k_{2}}{A_{1}} \frac{\partial A_{1}}{\partial x_{2}} u_{1}\right)\right. \\
& \left.\left.+\frac{k_{1} k_{2}}{A_{2}} \frac{\partial A_{2}}{\partial x_{1}} \frac{\partial u_{1}}{\partial x_{2}}-\frac{k_{1}^{2}}{A_{1} A_{2}} \frac{\partial A_{1}}{\partial x_{2}} \frac{\partial A_{2}}{\partial x_{1}} u_{1}\right]\right\}, \\
& =D_{n}\left\{\frac{\partial}{\partial x_{2}}\left(\frac{A_{1}}{A_{2}} \frac{\partial u_{2}}{\partial x_{2}}\right)-\frac{1}{A_{1} A_{2}}\left(\frac{\partial A_{1}}{\partial x_{2}}\right)^{2} u_{2}\right.
\end{aligned}
$$$$
+v\left[\frac{\partial}{\partial x_{2}}\left(\frac{1}{A_{2}} \frac{\partial A_{1}}{\partial x_{2}} u_{2}\right)-\frac{1}{A_{2}} \frac{\partial A_{1}}{\partial x_{2}} \frac{\partial u_{2}}{\partial x_{2}}\right]
$$$$
+\frac{1-v}{2}\left[\frac{A_{2}}{A_{1}} \frac{\partial A_{2}}{\partial x_{1}} \frac{\partial}{\partial x_{1}}\left(\frac{u_{2}}{A_{2}}\right)+\frac{\partial}{\partial x_{1}}\right.
$$$$
\times\left(\frac{A_{2}^{2}}{A_{1}} \frac{\partial}{\partial x_{1}}\left(\frac{u_{2}}{A_{2}}\right)\right)
$$$$
\left.-A_{1} A_{2} k_{2}^{2} u_{2}\right]
$$$$
+D_{m}\left[\frac{\partial}{\partial x_{1}}\left(\frac{k_{1} k_{2} A_{2}}{A_{1}} \frac{\partial u_{2}}{\partial x_{1}}\right)-\frac{\partial}{\partial x_{1}}\left(\frac{k_{2}^{2}}{A_{1}} \frac{\partial A_{2}}{\partial x_{1}} u_{2}\right)\right.
$$$$
\left.\left.-\frac{k_{1} k_{2}}{A_{1} A_{2}}\left(\frac{\partial A_{2}}{\partial x_{1}}\right)^{2} u_{2}+\frac{k_{1}^{2}}{A_{1}} \frac{\partial A_{2}}{\partial x_{1}} \frac{\partial u_{2}}{\partial x_{1}}\right]\right\},
$$

$L_{23}^{00} u_{3}$

$$
\begin{gathered}
=D_{n}\left[\frac{\partial}{\partial x_{2}}\left(A_{1}\left(k_{2}+v k_{1}\right) u_{3}\right)-\left(k_{1}+v k_{2}\right)\right. \\
\left.\times \frac{\partial A_{1}}{\partial x_{2}} u_{3}+\frac{1-v}{2} A_{1} k_{2} \frac{\partial u_{3}}{\partial x_{2}}\right],
\end{gathered}
$$

$L_{21}^{01} \gamma_{1}$

$$
\begin{aligned}
=D_{m}\left[\frac{\partial}{\partial x_{1}}\left(k_{2} \frac{\partial \gamma_{1}}{\partial x_{2}}\right)-\frac{\partial}{\partial x_{1}}\left(\frac{k_{2}}{A_{1}} \frac{\partial A_{1}}{\partial x_{2}} \gamma_{1}\right)\right. \\
\left.-\frac{k_{1}}{A_{1} A_{2}} \frac{\partial A_{1}}{\partial x_{2}} \frac{\partial A_{2}}{\partial x_{1}} \gamma_{1}+\frac{k_{1}}{A_{2}} \frac{\partial A_{2}}{\partial x_{1}} \frac{\partial \gamma_{1}}{\partial x_{2}}\right],
\end{aligned}
$$

$L_{22}^{01} \gamma_{2}$

$$
\begin{aligned}
=D_{m} & {\left[\frac{\partial}{\partial x_{1}}\left(\frac{A_{2} k_{2}}{A_{1}} \frac{\partial \gamma_{2}}{\partial x_{1}}\right)-\frac{\partial}{\partial x_{1}}\left(\frac{k_{2}}{A_{1}} \frac{\partial A_{2}}{\partial x_{1}} \gamma_{2}\right)\right.} \\
& \left.-\frac{k_{1}}{A_{1} A_{2}}\left(\frac{\partial A_{2}}{\partial x_{1}}\right)^{2} \gamma_{2}+\frac{k_{1}}{A_{1}} \frac{\partial A_{2}}{\partial x_{1}} \frac{\partial \gamma_{2}}{\partial x_{1}}\right] \\
+ & D_{n} A_{1} A_{2} k_{2} \gamma_{2}, \\
L_{31}^{00} u_{1} \quad & D_{n}\left[A_{2}\left(k_{1}+v k_{2}\right) \frac{\partial u_{1}}{\partial x_{1}}+\left(k_{2}+v k_{1}\right) \frac{\partial A_{2}}{\partial x_{1}} u_{1}\right. \\
& \left.+\frac{1-v}{2} \frac{\partial}{\partial x_{1}}\left(A_{2} k_{1} u_{1}\right)\right], \\
L_{32}^{00} u_{2} \quad & {\left[A_{1}\left(k_{2}+v k_{1}\right) \frac{\partial u_{2}}{\partial x_{2}}+\left(k_{1}+v k_{2}\right) \frac{\partial A_{1}}{\partial x_{2}} u_{2}\right.} \\
=D_{n} & \left.+\frac{1-v}{2} \frac{\partial}{\partial x_{2}}\left(A_{1} k_{2} u_{3}\right)\right],
\end{aligned}
$$




$$
\begin{aligned}
& L_{33}^{00} u_{3}=D_{n} A_{1} A_{2}\left[\frac{1-v}{2} \Delta u_{3}-\left(k_{1}^{2}+2 v k_{1} k_{2}+k_{2}^{2}\right) u_{3}\right], \quad L_{21}^{10} u_{1} \\
& L_{31}^{01} \gamma_{1}=D_{n} \frac{1-v}{2} \frac{\partial}{\partial x_{1}}\left(A_{2} \gamma_{1}\right) \\
& L_{32}^{01} \gamma_{2}=D_{n} \frac{1-v}{2} \frac{\partial}{\partial x_{2}}\left(A_{1} \gamma_{2}\right) \\
& L_{11}^{10} u_{1} \\
& =D_{m}(1-v)\left[\frac{\partial}{\partial x_{2}}\left(\frac{A_{1} k_{2}}{A_{2}} \frac{\partial u_{1}}{\partial x_{2}}\right)-\frac{\partial}{\partial x_{2}}\left(\frac{k_{1}}{A_{2}} \frac{\partial A_{1}}{\partial x_{2}} u_{1}\right)\right. \\
& \left.-\frac{k_{1}}{A_{1} A_{2}}\left(\frac{\partial A_{1}}{\partial x_{2}}\right)^{2} u_{1}+\frac{k_{2}}{A_{2}} A_{1} \frac{\partial A_{1}}{\partial x_{2}} \frac{\partial u_{1}}{\partial x_{2}}\right] \\
& +D_{n} A_{1} A_{2} k_{1} u_{1} \\
& L_{12}^{10} u_{2} \\
& =D_{n}(1-v)\left[\frac{\partial}{\partial x_{2}}\left(k_{1} \frac{\partial u_{2}}{\partial x_{1}}\right)-\frac{\partial}{\partial x_{2}}\left(\frac{k_{2}}{A_{1}} \frac{\partial A_{2}}{\partial x_{1}} u_{2}\right)\right. \\
& \left.-\frac{k_{2}}{A_{1} A_{2}} \frac{\partial A_{1}}{\partial x_{2}} \frac{\partial A_{2}}{\partial x_{1}} u_{2}+\frac{k_{1}}{A_{1}} A_{1} \frac{\partial A_{1}}{\partial x_{2}} \frac{\partial u_{2}}{\partial x_{1}}\right] \\
& L_{13}^{10} u_{3}=-D_{n} \frac{1-v}{2} A_{2} \frac{\partial u_{3}}{\partial x_{1}} \\
& L_{11}^{11} \gamma_{1} \\
& =D_{m}\left\{\frac{\partial}{\partial x_{1}}\left(\frac{A_{2}}{A_{1}} \frac{\partial \gamma_{1}}{\partial x_{1}}\right)-\frac{1}{A_{1} A_{2}}\left(\frac{\partial A_{2}}{\partial x_{1}}\right)^{2} \gamma_{1}\right. \\
& +v\left[\frac{\partial}{\partial x_{1}}\left(\frac{1}{A_{1}} \frac{\partial A_{2}}{\partial x_{1}} \gamma_{1}\right)-\frac{1}{A_{1}} \frac{\partial A_{2}}{\partial x_{1}} \frac{\partial \gamma_{1}}{\partial x_{1}}\right] \\
& +\frac{1-v}{2}\left[\frac{\partial}{\partial x_{2}}\left(\frac{A_{1}}{A_{2}} \frac{\partial \gamma_{1}}{\partial x_{2}}\right)+\frac{1}{A_{2}} \frac{\partial A_{1}}{\partial x_{2}} \frac{\partial \gamma_{1}}{\partial x_{2}}\right. \\
& -\frac{\partial}{\partial x_{2}}\left(\frac{1}{A_{2}} \frac{\partial A_{1}}{\partial x_{2}} \gamma_{1}\right) \\
& \left.\left.-\frac{1}{A_{1} A_{2}}\left(\frac{\partial A_{1}}{\partial x_{2}}\right)^{2} \gamma_{1}\right]\right\} \\
& +D_{n} \frac{1-v}{2} A_{1} A_{2} \gamma_{1} \text {, } \\
& L_{12}^{11} \gamma_{2} \\
& =D_{m}\left\{\frac{\partial}{\partial x_{1}}\left(\frac{1}{A_{1}} \frac{\partial A_{1}}{\partial x_{2}} \gamma_{2}\right)-\frac{1}{A_{2}} \frac{\partial A_{2}}{\partial x_{1}} \frac{\partial \gamma_{2}}{\partial x_{2}}\right. \\
& +v\left[\frac{\partial^{2} \gamma_{2}}{\partial x_{1} \partial x_{2}}-\frac{1}{A_{1} A_{2}} \frac{\partial A_{1}}{\partial x_{2}} \frac{\partial A_{2}}{\partial x_{1}} \gamma_{2}\right] \\
& +\frac{1-v}{2}\left[\frac{\partial^{2} \gamma_{2}}{\partial x_{1} \partial x_{2}}+\frac{1}{A_{1}} \frac{\partial A_{1}}{\partial x_{2}} \frac{\partial \gamma_{2}}{\partial x_{1}}\right. \\
& -\frac{\partial}{\partial x_{2}}\left(\frac{1}{A_{2}} \frac{\partial A_{2}}{\partial x_{1}} \gamma_{2}\right) \\
& \left.\left.-\frac{1}{A_{1} A_{2}} \frac{\partial A_{1}}{\partial x_{2}} \frac{\partial A_{2}}{\partial x_{1}} \gamma_{2}\right]\right\} \text {, } \\
& =D_{m} \frac{1-v}{2}\left[\frac{\partial}{\partial x_{1}}\left(k_{2} \frac{\partial u_{1}}{\partial x_{2}}\right)-\frac{\partial}{\partial x_{1}}\left(\frac{k_{1}}{A_{1}} \frac{\partial A_{1}}{\partial x_{2}} u_{1}\right)\right. \\
& \left.-\frac{k_{1}}{A_{1} A_{2}} \frac{\partial A_{1}}{\partial x_{2}} \frac{\partial A_{2}}{\partial x_{1}} u_{1}+\frac{k_{2}}{A_{2}} \frac{\partial A_{2}}{\partial x_{1}} \frac{\partial u_{1}}{\partial x_{2}}\right], \\
& =D_{m} \frac{1-v}{2}\left[\frac{\partial}{\partial x_{1}}\left(\frac{A_{2} k_{1}}{A_{1}} \frac{\partial u_{2}}{\partial x_{1}}\right)-\frac{\partial}{\partial x_{1}}\left(\frac{k_{2}}{A_{1}} \frac{\partial A_{2}}{\partial x_{1}} u_{2}\right)\right. \\
& \left.-\frac{k_{2}}{A_{1} A_{2}} \frac{\partial A_{2}}{\partial x_{1}} u_{2}+\frac{k_{1}}{A_{1}} \frac{\partial A_{2}}{\partial x_{1}} \frac{\partial u_{2}}{\partial x_{1}}\right] \\
& +D_{n} \frac{1-v}{2} A_{1} A_{2} k_{2} u_{2} \text {, } \\
& L_{23}^{10} u_{3}=-D_{n} \frac{1-v}{2} A_{1} \frac{\partial u_{3}}{\partial x_{2}}, \\
& =D_{m}\left\{\frac{\partial}{\partial x_{2}}\left(\frac{1}{A_{2}} \frac{\partial A_{2}}{\partial x_{1}} \gamma_{1}\right)-\frac{1}{A_{1}} \frac{\partial A_{1}}{\partial x_{2}} \frac{\partial \gamma_{1}}{\partial x_{1}}\right. \\
& +v\left[\frac{\partial^{2} \gamma_{1}}{\partial x_{1} \partial x_{2}}-\frac{1}{A_{1} A_{2}} \frac{\partial A_{1}}{\partial x_{2}} \frac{\partial A_{2}}{\partial x_{1}} \gamma_{1}\right] \\
& +\frac{1-v}{2} \\
& \times\left[\frac{\partial^{2} \gamma_{1}}{\partial x_{1} \partial x_{2}}+\frac{1}{A_{2}} \frac{\partial A_{2}}{\partial x_{1}} \frac{\partial \gamma_{1}}{\partial x_{2}}\right. \\
& \left.\left.-\frac{\partial}{\partial x_{1}}\left(\frac{1}{A_{1}} \frac{\partial A_{1}}{\partial x_{2}} \gamma_{1}\right)-\frac{1}{A_{1} A_{2}} \frac{\partial A_{1}}{\partial x_{2}} \frac{\partial A_{2}}{\partial x_{1}} \gamma_{1}\right]\right\}, \\
& =D_{m}\left\{\frac{\partial}{\partial x_{2}}\left(\frac{A_{1}}{A_{2}} \frac{\partial \gamma_{2}}{\partial x_{2}}\right)-\frac{1}{A_{1} A_{2}}\left(\frac{\partial A_{1}}{\partial x_{2}}\right)^{2} \gamma_{2}\right. \\
& +v\left[\frac{\partial}{\partial x_{2}}\left(\frac{1}{A_{2}} \frac{\partial A_{1}}{\partial x_{2}} \gamma_{2}\right)-\frac{1}{A_{2}} \frac{\partial A_{1}}{\partial x_{2}} \frac{\partial \gamma_{2}}{\partial x_{2}}\right] \\
& +\frac{1-v}{2} \\
& \times\left[\frac{\partial}{\partial x_{1}}\left(\frac{A_{2}}{A_{1}} \frac{\partial \gamma_{2}}{\partial x_{1}}\right)+\frac{1}{A_{1}} \frac{\partial A_{2}}{\partial x_{1}} \frac{\partial \gamma_{2}}{\partial x_{1}}\right. \\
& \left.\left.-\frac{\partial}{\partial x_{1}}\left(\frac{1}{A_{1}} \frac{\partial A_{2}}{\partial x_{1}} \gamma_{2}\right)-\frac{1}{A_{1} A_{2}}\left(\frac{\partial A_{2}}{\partial x_{1}}\right)^{2} \gamma_{2}\right]\right\} \\
& +D_{n} \frac{1-v}{2} A_{1} A_{2} \gamma_{2} \text {. }
\end{aligned}
$$




\section{Acknowledgment}

This work was supported by the Committee of Science and Technology of Mexico (CONACYT) through the research Grants (Project no. 000000000101415), which is gratefully acknowledged.

\section{References}

[1] H. L. Duan, J. Wang, and B. L. Karihaloo, "Theory of elasticity at the nanoscale," Advances in Applied Mechanics, vol. 42, pp. 1-68, 2009.

[2] H. Altenbach and V. A. Eremeyev, "On the shell theory on the nanoscale with surface stresses," International Journal of Engineering Science, vol. 49, pp. 1294-1301, 2011.

[3] S. Lekhnitskii, Anisotropic Plates, Routledge, 1968.

[4] P. M. Naghdi, The Theory of Plates and Shells, Springer, Berlin, Germany, 1972.

[5] V. V. Novozhilov, Thin Shell Theory, Springer, New York, NY, USA, 2nd edition, 1971.

[6] S. Timoshenko and S. Woinowsky-Krieger, Theory of Plates and Shells, McGraw-Hill, New York, NY, USA, 1959.

[7] K. D. Mindlin, An Introduction to the Mathematical Theory of Vibrations of Elastic Plates, World Scientific, 2006.

[8] I. Y. Khoma, Generalized Theory of Anisotropic Shells, Naukova dumka, Kiev, Russia, 1987.

[9] B. L. Pelekh, The Generalized Theory of Shells, L'vov University Press, L'vov, Ukraine, 1978.

[10] J. N. Reddy, Mechanics of Laminated Composite Plates and Shells: Theory and Analysis, CRC Press LLC, Boca Raton, Fla, USA, 2nd edition, 2004.

[11] N. A. Kil'chevskii, Fundamentals of the Analytical Mechanics of Shells, House of ANUkrSSR, Kiev, Russia, 1963.

[12] I. N. Vekua, Shell Theory, General Methods of Construction, Pitman Advanced Pub. Program, Boston, Mass, USA, 1986.

[13] V. I. Gulyaev, V. A. Bazhenov, and P. P. Lizunov, The Nonclassical Theory of Shells and Its Application to the Solution of Engineering Problems, Vyshcha Shkola, L'vov, Ukraine, 1978.

[14] B. L. Pelekh and M. A. Shorol'skii, Contact Problems of the Theory of Elastic Anisotropic Shells, Naukova dumka, Kiev, Russia, 1980.

[15] B. L. Pelekh and V. A. Lazko, Laminated Anisotropic Plates and Shells with Stress Concentrators, Naukova dumka, Kiev, Russia, 1982.

[16] B. Y. Kantor and V. V. Zozulya, "Connected problem on contact plate with rigid body though the heat-conducting layer," Doklady Akademii Nauk Ukrainskoy SSR, pp. 31-33, 1988.

[17] V. V. Zozulya, "Contact cylindrical shell with a rigid body though the heat-conducting layer," Doklady Akademii Nauk Ukrainskoy SSR, pp. 48-51, 1989.

[18] V. V. Zozulya and Y. N. Borodenko, "Connecting problem on contact of cylindrical shells with a rigid body in temperature though the heat-conducting layer," Doklady Akademii Nauk Ukrainskoy SSR, pp. 35-41, 1992.

[19] V. V. Zozulya, "The combined problem of thermoelastic contact between two plates through a heat conducting layer," Journal of Applied Mathematics and Mechanics, vol. 53, no. 5, pp. 622-627, 1989.

[20] V. V. Zozulya, "Contact cylindrical shell with a rigid body though the heat-conducting layer in transitional temperature field," Mechanics of Solids, vol. 2, pp. 160-165, 1991.
[21] V. V. Zozulya, "Contact of a shell and rigid body though the heat-conducting layer temperature field," International Journal of Mathematics and Computers in Simulation, vol. 2, pp. 138-145, 2007.

[22] V. V. Zozulya, "Contact of the thin-walled structures and rigid body though the heatconducting layer," in Theoretical and Experimental Aspects of Heat and Mass Transfer, J. Krope, S. H. Sohrab, and F.-K. Benra, Eds., pp. 145-150, WSEAS Press, Acapulco, Mexico, 2008.

[23] V. V. Zozulya, "Heat transfer between shell and rigid body through the thin heat-conducting layer taking into account mechanical contact," WIT Transactions on Engineering Sciences, vol. 61, pp. 81-90, 2008.

[24] V. V. Zozulya and M. Aguilar, "Thermo-elastic contact and heat transfer between plates and shells through the heat-conducting layer," Computat Studies, vol. 3, pp. 85-94, 2000.

[25] V. V. Zozulya and Y. N. Borodenko, "Thermoplastic contact of rigidly fixed shell with a rigid body though the heat-conducting layer," Doklady Akademii Nauk Ukrainskoy SSR, pp. 47-53, 1991.

[26] V. V. Zozulya, "Stress intensity factor in a contact problem for a plane crack under an antiplane shear wave," International Applied Mechanics, vol. 43, no. 9, pp. 1043-1047, 2007.

[27] V. V. Zozulya, "Contact of the thin-walled structures and rigid body though the heatconducting layer," in Theoretical and Experimental Aspects of Heat and Mass Transfer, J. Krope, S. H. Sohrab, and F.-K. Benra, Eds., pp. 145-150, WSEAS Press, Acapulco, Mexico, 2008.

[28] V. V. Zozulya, "A high-order theory for functionally graded axially symmetric cylindrical shells," Archive of Applied Mechanics, vol. 83, no. 3, pp. 331-343, 2013.

[29] V. V. Zozulya, "A high order theory for functionally graded shell," World Academy of Science, Engineering and Technology, vol. 59, pp. 779-784, 2011.

[30] A. K. Noor, "Bibliography of monographs and surveys on shells," Applied Mechanics Reviews, vol. 43, no. 9, pp. 223-234, 1990.

[31] W. Pietraszkiewicz, "Addendum to: bibliography of monographs and surveys on shells," Applied Mechanics Reviews, vol. 45 , no. 6, p. 269, 1992.

[32] H. Altenbach and V. A. Eremeev, Shell Like Structures. Non Classical Theories and Applications, Springer, New York, NY, USA, 2011.

[33] W. T. Koiter, Foundations and Basic Equations of Shell Theory. A Survey of Recent Progress, Springer, New York, NY, USA, 2nd edition, 1969.

[34] H.-S. Shen, Functionally Graded Materials: Nonlinear Analysis of Plates and Shells, CRC Press, Taylor \& Francis, Boca Raton, Fla, USA, 2009. 

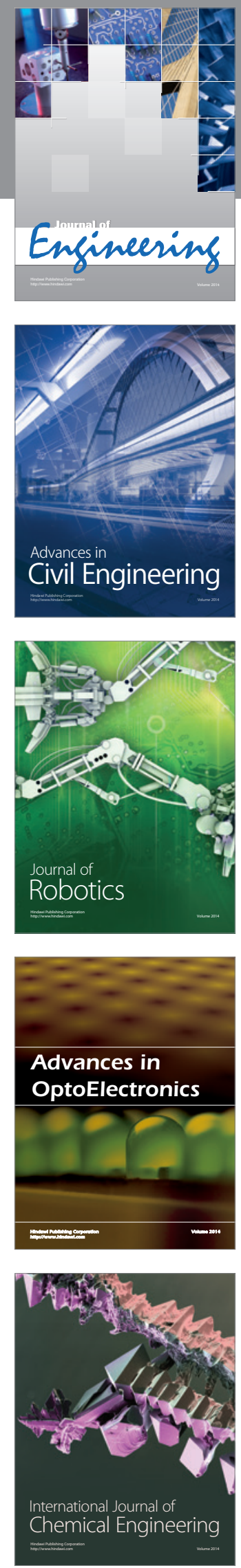

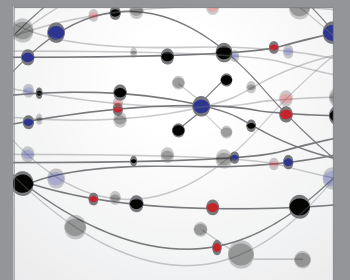

The Scientific World Journal
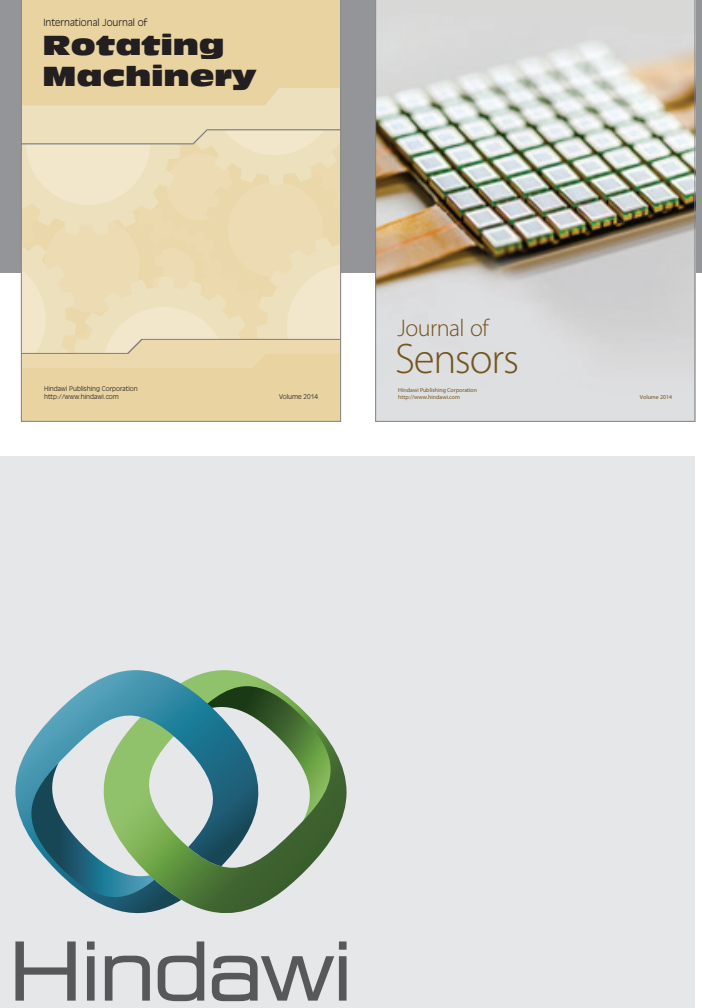

Submit your manuscripts at http://www.hindawi.com
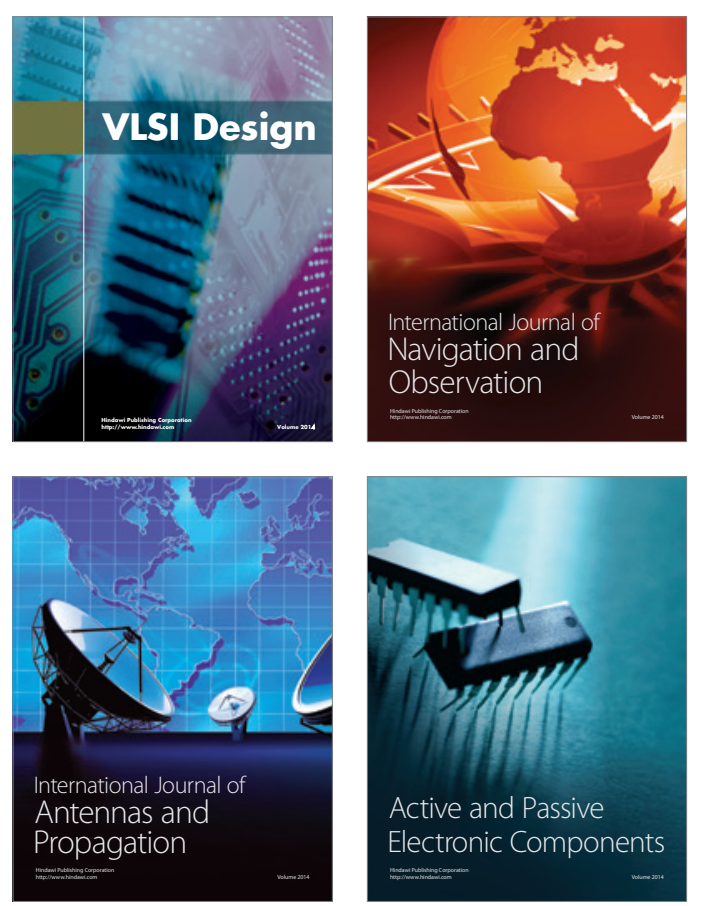
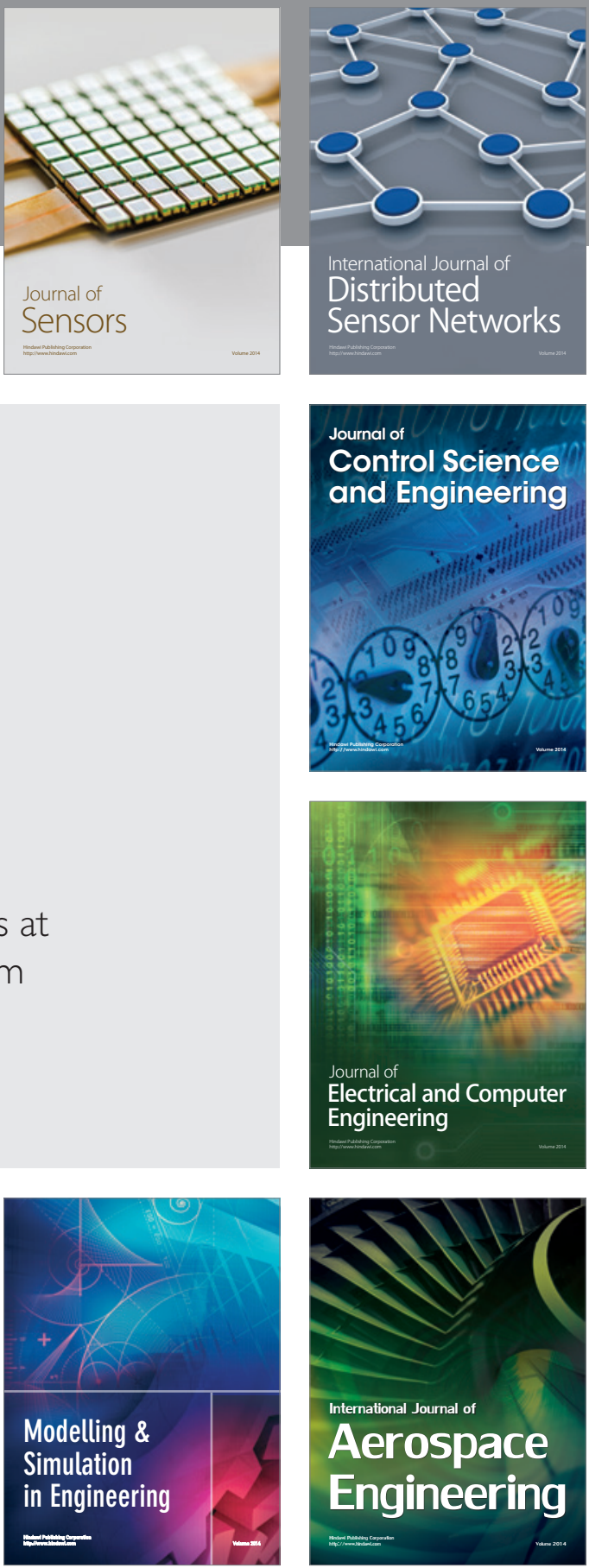

Journal of

Control Science

and Engineering
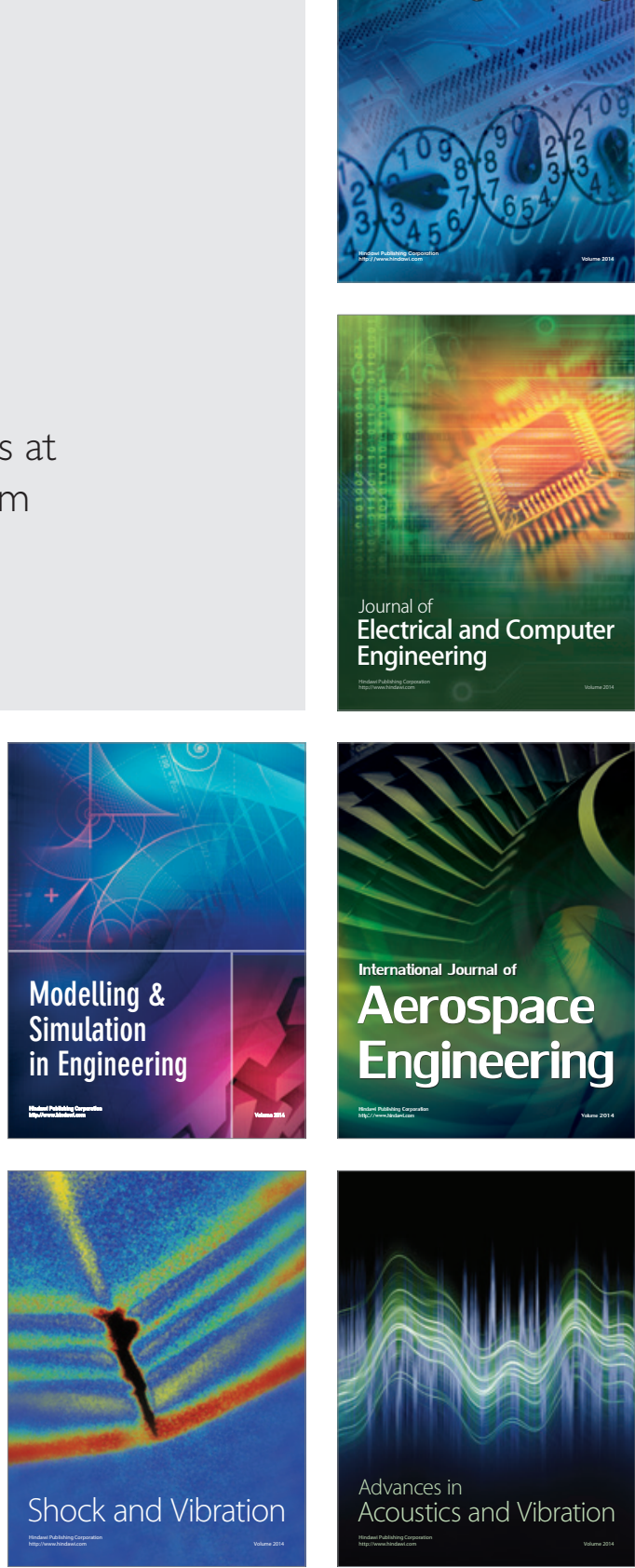